\title{
A Column-Generation and Branch-and-Cut Approach to the Bandwidth-Packing Problem
}

\begin{tabular}{|c|c|}
\hline Volume 111 & Number 2 \\
\hline $\begin{array}{l}\text { Christine Villa and Karla } \\
\text { Hoffman } \\
\text { George Mason University } \\
\text { Mail Stop 4A6 } \\
\text { Systems Engineering and } \\
\text { Operations Research Department } \\
4400 \text { University Drive, } \\
\text { Fairfax, VA } 22030\end{array}$ & $\begin{array}{l}\text { The telecommunications problem of } \\
\text { assigning calls with point to point demand } \\
\text { to a capacitated network where each call } \\
\text { can be assigned to at most one path has } \\
\text { been called the Bandwidth-Packing } \\
\text { Problem. For a given network, with } \\
\text { specified arc costs and arc capacities, one } \\
\text { wishes to route calls (defined by a starting } \\
\text { and ending point) through the network to } \\
\text { maximize the profit from the calls routed. } \\
\text { Each such call is single path routed and } \\
\text { not all calls will be routed. We propose a } \\
\text { branch-and-cut methodology coupled with } \\
\text { column generation to optimally solve such } \\
\text { problems. We examine the alternative } \\
\text { approaches in the literature and explain } \\
\text { how this new method takes the best of } \\
\text { all components of methods suggested } \\
\text { previously. The method we suggest } \\
\text { is new in that it includes a linear } \\
\text { programming-based heuristic for obtaining } \\
\text { good lower bounds, uses lifted minimal } \\
\text { covers that take into account special- } \\
\text { ordered set constraints, and dynamically } \\
\text { choose among three alternative branching } \\
\text { strategies. In addition, whenever a new }\end{array}$ \\
\hline
\end{tabular}

\begin{abstract}
column is generated, it is lifted into all existing cuts. We also discuss the need to generate all tied optimal linear optimization solutions if one wishes to assure that the solution obtained is optimal. Our computational results provide solutions to problems previously unsolvable.
\end{abstract}

Key words: capacitated networks; column generation; combinatorial optimization; cutting planes; facet-defining cuts; heuristics; integer programming; network planning; telecommunications networks; zero-one optimization.

Accepted: December 14, 2005

Available online: http://www.nist.gov/jres

\section{Introduction}

The bandwidth-packing problem is a combinatorial optimization problem arising from telecommunication networks where demand exceeds capacity and where point-to-point calls with varying bandwidths are routed on this network such that each call uses a single path. These problems occur within ATM technology and when video data is routed. It belongs to the broad class of multi-commodity flow problems that are commonly associated with communication, computer, transportation, and distribution network applications. Calls between pairs of nodes define the commodities, and links connecting nodes represent transmission lines in the telecomm network. Given a set of calls with their revenue and bandwidth requirements and an undirected network with its fixed arc/link capacities and costs, the problem is to assign calls from a request table to paths such that network capacities are not violated and profit is maximized.

When one can split a call among a variety of paths, then the problem can be solved by linear programming techniques. However, there are many telecommunications problems (as well as applications out- 
side telecommunications, see Barnhart et al. [2000] ) where the commodity must be routed on a single path. This additional requirement makes the problem NP hard, and few papers address methodology to tackle this problem. We present a technique that has become popular recently for difficult, large 0-1 problems: that of performing both column-generation and row-generation within a tree-search. We also include a heuristic to improve bounds on the problem.

\subsection{Problem Formulation}

The bandwidth-packing problem (BWP) can be formulated as a $0-1$ integer-programming problem over a network defined by nodes $i$ and arcs or links $l$ that connect such nodes. A path consists of a route for a given call $i$ through a collection of links that connects a source node $s_{i}$ to terminal node $t_{i}$. The objective function is to maximize profit and the decisions to be made are whether to route a given call and if routed, to determine which of the multiple paths to pick to route the call.

Let the 0-1 decision variable $x_{i j}$ indicate whether call $i$ is routed on path $j$. Then the objective is to maximize profit from assigning calls to paths, such that calls are assigned at most once and link capacities are not violated. The profit of path $j$ is defined as the revenue for call $i, r_{i}$, minus the total bandwidth cost of using the links in path $j$ (i.e., the profit of path $j$ associated with call $i$ is equal to: $r_{i}-d_{i} \sum_{l=1}^{m} \delta_{l_{j}} c_{l}$ ) where $\delta_{l_{j}}$ is an indicator that equals one whenever link $l$ is used in path $j, d_{i}$ is the bandwith requirement (demand) of call $i$, and $c_{l}$ is the per unit bandwidth cost of using link $l$. We assume that there are $n$ calls and $m$ links in the network.

Telecommunications Network

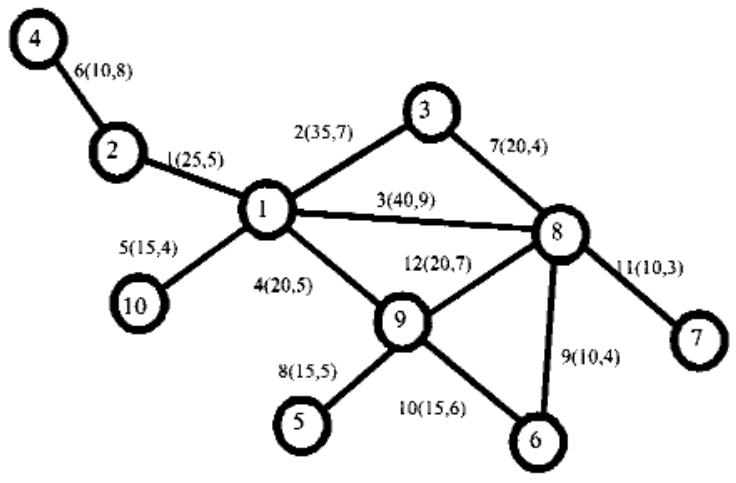

Link (capacity, cost)
Then with the following notational definitions:

Call $i: \quad r_{i} \quad$ revenue for call $i$

$d_{i}$ bandwidth requirement (demand) of call $i$

$s_{i}, t_{i}$ source node and terminal node of call $i$

$P_{i}$ the set of paths for call $i$

Link $l$ : $\quad$ capacity of link $l$, in bandwidth

$c_{i}$ unit cost of bandwidth on link $l$.

Variable: $x_{i j} l$ if call $i$ is assigned to path $j ; 0$ otherwise.

We obtain the integer linear optimization problem:

$$
\max \sum_{i-1, \cdots, n} \sum_{j \in P_{i}}\left[r_{i}-d_{i} \sum_{l-1, \cdots, m} \delta_{l_{j}} c_{l}\right] x_{i j}
$$

Subject to :

$$
\begin{array}{cc}
\sum_{j \in S_{i}} x_{i j} \leq 1 & \forall i=1, \ldots, n \in \text { calls -- } \\
& \text { routing constraints (SOS) } \\
\sum_{i=1, \cdots, n} \sum_{j \in P_{i}} \delta_{l_{j}} d_{i} x_{i j} \leq b_{l} & \forall 1=1, \ldots, m \in \text { links -- } \\
\text { capacity constraints (KNAPSACKS) } & \\
x_{i j \in\{}\{0,1\} & \forall i=1, \ldots ., n, j \in P_{i}
\end{array}
$$

The first set of constraints insures that each call is either routed or not, grouping the paths by call into nonoverlapping special-order sets. The second set of constraints is a collection of knapsack constraints that insure no link capacities are violated. All variables for a given call share a single coefficient (the bandwidth requirement for the call) for each knapsack constraint. (Note that $x_{i j}=1$ is implied by the routing constraints, so explicitly setting the upper bound to one is not necessary.)

\subsection{A Small Example}

We illustrate the problem structure with the following problem (Fig. 1).

$$
\begin{array}{ll}
\mathrm{si}=\text { source node } & \mathrm{ti}=\text { terminal node } \\
\mathrm{di}=\text { bandwidth demand } & \mathrm{r}=\text { revenue }
\end{array}
$$

\begin{tabular}{cllcccccc}
\multicolumn{7}{c}{ Call Table } \\
\hline \hline Call & si/ti & di & ri & Call & si/ti & di & ri \\
\hline 1 & $1 / 3$ & 10 & 420 & $\mid$ & 11 & $4 / 6$ & 6 & 850 \\
2 & $1 / 4$ & 7 & 380 & $\mid$ & 12 & $6 / 3$ & 3 & 200 \\
3 & $1 / 10$ & 6 & 400 & $\mid$ & 13 & $7 / 10$ & 5 & 370 \\
4 & $1 /$ & 6 & 390 & $\mid$ & 14 & $8 / 2$ & 6 & 500 \\
5 & $2 / 7$ & 5 & 500 & $\mid$ & 15 & $8 / 10$ & 5 & 340 \\
6 & $2 / 6$ & 5 & 490 & $\mid$ & 16 & $8 / 5$ & 2 & 120 \\
7 & $2 / 5$ & 7 & 400 & $\mid$ & 17 & $9 / 2$ & 6 & 460 \\
8 & $3 / 10$ & 2 & 150 & $\mid$ & 18 & $9 / 3$ & 8 & 450 \\
9 & $3 / 4$ & 4 & 450 & $\mid$ & 19 & $10 / 6$ & 5 & 360 \\
10 & $3 / 5$ & 8 & 500 & $\mid$ & 20 & $10 / 2$ & 5 & 170
\end{tabular}

Fig. 1. A Sample Telecommunications Network with Node Numbers, Link Numbers, Link Capacities and Link Costs Identified. A sample call table is also given. 
This problem (Fig. 1) then translates to the following A-matrix structure (Table 1) where there are a collection of special-ordered set constraints and a set of knapsack constraints, where in each knapsack, all paths (variables) associated with the call have the same coefficient. The reason for this commonality is that, for a given call, each of the paths uses the same amount of bandwidth. In table 1 we note that paths P1 through P4 relate to call 1, paths P5 through P10 relate to Call 18 and paths P11 through P14 relate to call 14.

In this paper, we test our approach using the standard data sets used in earlier work. All problems have the same basic two data sets: a call table and a link table. The call table lists the call's source and destination nodes, its demand, and its revenue in units of bandwidth. The link table specifies for each link its adjoining nodes, its bandwidth capacity, and the cost per unit of bandwidth using the link. We show the paths related to only three calls (call 1: (node 1 to node 3 ), call 18 (9 to 3 ) and call 14 (8 to 2)) of this problem to illustrate the structure of the A-matrix.

Since there is no requirement to route a call in this formulation, only profitable paths need to be considered. While the number of nodes in the network typically will not be large ( $<40$ nodes in our test problems), the number of profitable paths increases quickly with the number of links.

Parker and Ryan (1994) showed the bandwidthpacking problem is NP-Hard since it contains a number of 0-1 knapsack constraints as part of the entire constraint set.
Even for small networks, the number of possible paths generated for each call through pure enumeration can be enormous. Incurring this upfront cost of generating all such paths when most will not have any possibility of being in the optimal solution is unnecessary. Instead, one prefers to generate columns only as needed, that is, only when they will improve the lp-solution. This approach is known as "column generation" and requires a "pricing" algorithm that either generates such a column or proves that no such column exists. Since the linear programming solution serves as an upper bound on the solution value, one wants this upper bound to be as close to the true integer solution as possible. One can tighten the linear programming relaxation by applying cutting planes that more closely approximate the convex hull of the feasible integer points.

Given a good upper bound on the solution value, one must also have a good lower bound on the solution for a bounding procedure such as branch-and-bound to work efficiently. We employ an lp-based heuristic for obtaining good lower bounds on the problem. This paper will present a solution methodology that incorporates all of these techniques and uses a dynamic branching strategy based on setting many variables simultaneously to zero, to improve the overall search. We begin by summarizing the prior research on this problem.

Table 1. A-Matrix for the sample problem in Fig. 1

\begin{tabular}{|c|c|c|c|c|c|c|c|c|c|c|c|c|c|c|c|}
\hline \multirow[t]{2}{*}{ Link } & \multicolumn{14}{|c|}{ Paths } & \multirow[t]{2}{*}{ RHS } \\
\hline & $\mathrm{P} 1$ & P2 & P3 & P4 & P5 & P6 & P7 & P8 & P9 & P10 & P11 & P12 & P13 & P14 & \\
\hline SOS1 & 1 & 1 & 1 & 1 & & & & & & & & & & & 1 \\
\hline SOS2 & & & & & 1 & 1 & 1 & 1 & 1 & 1 & & & & & 1 \\
\hline SOS3 & & & & & & & & & & & 1 & 1 & 1 & 1 & 1 \\
\hline$(1,8)$ & & 10 & & & & & 8 & & & 8 & & 6 & & & 40 \\
\hline$(1,2)$ & & & & & & & & & & & 6 & 6 & 6 & 6 & 25 \\
\hline$(1,9)$ & & & 10 & 10 & & 8 & & & & & & & 6 & 6 & 20 \\
\hline$(3,8)$ & & 10 & 10 & 10 & 8 & & & 8 & 8 & & 6 & & & & 20 \\
\hline$(9,8)$ & & & & 10 & 8 & & 8 & 8 & & & & & 6 & & 20 \\
\hline$(9,6)$ & & & 10 & & & & & & 8 & 8 & & & & 6 & 15 \\
\hline$(6,8)$ & & & 10 & & & & & & 8 & 8 & & & & 6 & 10 \\
\hline
\end{tabular}




\subsection{Previous Work on This Problem}

Initial work on the bandwidth-packing problem focused on heuristic methods. Cox et al. (1991) described the problem for dynamic call routing where the bandwidth-packing problem is solved as a subproblem. They did not envision the number of nodes in the network to be large ( 30 to 50 nodes) but the number of calls could be as high as 100 . Even in sparse networks, the number of different paths possible for a single call can be quite large. Also, the number of possible routes increases with the density of the network. Cox et al. used a genetic algorithm and permutation based approach to path assignment. Each potential solution is represented as a permutation of an ordering of the calls and is evaluated by assigning the shortest path available after previous calls in the ordering are routed. Anderson et al. (1993) also used a permutation approach but within a tabu search. Laguna and Glover (1993) developed a non-permutation tabu search algorithm, where potential solutions are evaluations of changes in path assignment for a given call. Gavish and Altinkemer (1990) and Fischer et al. (1994) used Lagrangian Relaxation to obtain good feasible solutions to special cases of the bandwidth-packing problem. Amiri and Barkhi (1999) consider a multi-hour bandwidth-packing problem and also use Lagrangian Relaxation to obtain heuristic solutions to their problems. A number of recent papers have examined how to assign calls to links to minimize the queueing delay when arrivals are stochastic: see Rolland et al. (1999) and Amiri et al. (1999).

Since our paper is concerned with proving optimality to difficult bandwidth-packing problems, we spend more time examining the prior work on exact methods. Parker and Ryan (1994) describe a column-generation branch-and-bound procedure (also known as branch and price).

The problem was formulated as an integer programming problem by applying column generation based on the linear programming relaxation imbedded in a branch and bound procedure. Their branching strategy chooses a certain fractional path for a given call. They then create $k+1$ branches. On $k$ of these branches, they stipulate that the call cannot use arc/link $j(j=1 \ldots k)$. The $k+1$ st leaf requires that the call not use any of the arcs/links. They dynamically set the optimality tolerance and solve all but two instances of their problem set within $95 \%$ of optimality. They prove optimality to only two of these fourteen problems, however. Parker and Ryan do not use cutting planes.

Park et al. (1996) embed cutting planes based on lifted minimal covers (see Padberg 1975) within column generation to create the $l p$-relaxation, and incorporate these cuts into a branch-and-cut procedure. Cuts were not generated within the tree unless the branch-andprice routines failed to prove optimality within a reasonable period of time. Then, cutting plane routines were also employed in the branching tree. The authors employed a traditional $x_{i j}=0 / 1$ branching strategy; however, for comparison they implemented a simplified version of Parker and Ryan's approach and showed that the use of cutting planes substantially improves solution times for either branching rule. We note that the test-set of problems used by Park et al. were randomly generated problems and appear to be much easier to solve than those presented in the Parker and Ryan or those in the Glover and Laguna papers.

Recently, two other papers use a branch-cut-andprice algorithm for the bandwidth-packing problem. Barnhart et al. (2000) again use lifted minimal covers within a branch-cut-and-price approach. Their branching strategy is different from that of either Park et al. or Parker and Ryan. They identify a call that is split between two paths and then identify the node such that prior to that node the two paths are identical, and call this sub-path $s$. They call this node the divergence node, labeled $d$, and the two arcs that cause the divergence $a_{1}$ and $a_{2}$. They partition all arcs leading from divergent node $d$ into two disjoint partitions such that one set $\mathrm{A}_{1}$ contains $a_{1}$ and the other set $\mathrm{A}_{2}$ contains $a_{2}$. On one branch all paths that contain subpath $s$ and any arc in $\mathrm{A}_{1}$ are set to zero and on the other branch all paths that contain subpath $s$ and any arc in $\mathrm{A}_{2}$ are set to zero. Thus, this branching approach has two properties: (1) it is easy to generate new paths for a call without changing the pricing of new columns and (2) many variables (paths) might be fixed to zero on a single branch. They use a depth-first tree search in order to obtain good feasible solutions early in the search. They discovered that on many branches the objective function changed little because of the symmetry in the problem. They therefore incorporated lifted cover inequalities and perform this lifting based on the algorithm of $\mathrm{Gu}$ et al. (1995). We note that the authors do not use the special-ordered sets to strengthen these cuts. The success of these authors was impetus for us to see if providing stronger cuts and providing a heuristic might improve upon their results.

Finally, Geffard (2001) also uses a branch-cut-andprice approach. Here, the minimal covers are not lifted but a heuristic is used to obtain good bounds. The authors branch on arcs for a specific call, similar to one of the three branching strategies that are employed in this paper. We will discuss this branching strategy later. 
Their testing showed that using a heuristic enhanced the performance of their code significantly.

\subsection{Contributions of This Paper}

In our view, the contributions of this paper include:

(1) the incorporation of much stronger cuts into a branch-cut-and-price code for the bandwidthpacking problem. These lifted minimal covers with special-ordered sets are easily incorporated into the pricing algorithm and, whenever a new path is generated for a given call that uses a link not previously used by any paths for that call, the variable (path) is lifted into all cuts associated with that link. This lifting calculation is inexpensive to perform.

(2) the inclusion of a dynamic branching strategy that does not require significant changes to the pricing algorithm.

(3) the inclusion of an $l p$-based heuristic that quickly finds very good solutions and is capable of being used throughout the tree.

(4) the solution of previously unsolved problems in the standard test-set of bandwidth-packing problems.

(5) a small example that illustrates the need for generating all optimal solutions to the linear programming problems on each branch.

\subsection{Outline}

The remainder of this paper is organized as follows. Section 2 describes each sub-algorithm of the branchcut-and-price code. Section 3 provides computational results on two published sets 13 of test problems. Section 4 provides conclusions and avenues for future research.

\section{Solution Methodology}

Since pricing and cutting are complementary procedures for tightening an $l p$-relaxation, our method generates both columns and cuts throughout a branch and bound tree. Because there are too many possible columns to handle efficiently and most of them will have their associated variable equal to zero in an optimal solution, a more efficient approach than enumeration is to start with only a small subset of the feasible columns and then add others as needed. This process is called "pricing" and uses reduced-cost information to add columns only when they can improve the linear programming solution value.

Likewise, as the above discussion has highlighted, adding cutting planes based on the polyhedral structure of the $0-1$ polyhedron is a very efficient way of tightening the linear programming approximation to the integer-programming problem.

In addition, we add an $l p$-based bounding heuristic to obtain a good ip-lower bound early and we attempt to update this bound whenever columns and cuts are generated. Having both a good upper bound and a good lower bound allows variables to be fixed based on the "gap" (i.e. the difference between the $l p$-solution and the best-known feasible solution).

The basic algorithm begins by solving the lp-relaxation of the BWP problem with only a subset of paths $\mathrm{S}_{i}-\mathrm{P}_{i}$ per call. Then, for each call, we generate columns (paths) based on dual prices. When no additional columns will improve the $l p$-solution, we begin generating cutting planes for each link constraint coupled with the entire set of special-ordered set (SOS) constraints. These cutting planes are considerably stronger than those generated when the SOS constraints are ignored. We continue cycling through cut generation and column generation until we can find no additional cuts or columns to improve the $l p$-solution. At this point, we perform tree search where at each branch of the tree, we again employ both column and cut generation. In addition, we employ an $l p$-based heuristic within the branching tree. Figure 1 presents the overall algorithm. We present each of the sub-algorithms below.

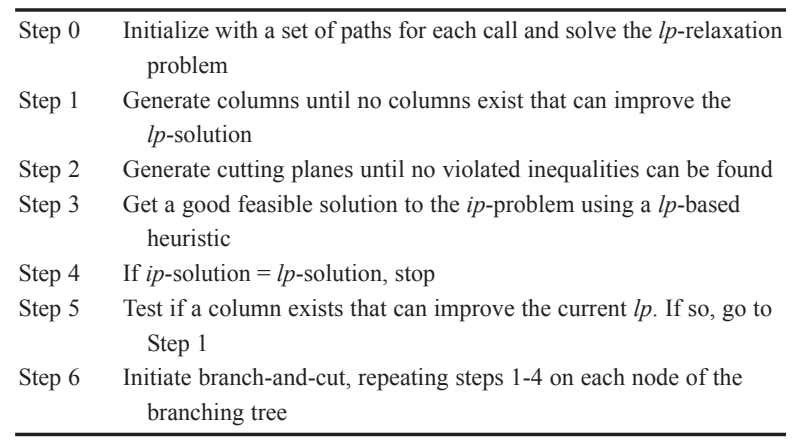

Fig. 2. Column Generation/Branch \& Cut Algorithm for BWP Problem

\subsection{Solving the First Linear Program}

Our approach to generating columns is similar to that of prior authors. For the first linear program, we use the cost on the links to determine at least one shortest path for each call. Where the solution to the shortest path 
problem is not unique, we generate all shortest paths. For data sets where the link costs were nonzero, there was usually only one or two optimal paths per call. However, for data sets where the link costs were all zero, generating all shortest paths generated many per call, so we only added up to 5 paths (columns) per call in the initial formulation of the linear program. However, prior to branching, we added all paths of a call that had a reduced cost of zero. This approach is different from Parker and Ryan who use only one shortest path for the problem, or Park et al. who use a k-shortest path algorithm (but in most of their paper report test results for $\mathrm{k}$ set equal to 1). Given at least one path for each call, we solve the first $l p$. We now present the general pricing algorithm which is performed after every $l p$ call.

\subsection{Generating Columns}

By $l p$-duality theory, the $l p$-solution is optimal if and only if the dual solution is feasible. If any of the dual constraints are not satisfied for any call, then there exists a column or columns that would have been in the basis had they been in the $l p$-relaxation. We use the same column generation scheme as Parker and Ryan. It is briefly described here. Let $y_{i}$ denote the dual variables corresponding to the routing constraints, $z_{l}$ and the dual variables corresponding to the capacity constraints $y_{i}$, and let $S_{i}$ denote the current subset of paths in the $l p$-relaxation. Call this problem $\mathrm{LP}^{\mathrm{S}}$.

$\max \sum_{i=1, \cdots, n} \sum_{j \in P_{i}}\left[r_{i}-d_{i} \sum_{l-1, \cdots, m} \delta_{l_{j}} c_{l}\right] x_{i j}$

Subject to :

$y_{i}: \sum_{j \in S_{i}} x_{i j} \leq 1$

$\forall i=1, \ldots, n \in$ calls --

routing constraints (SOS)

$z_{l}: \sum_{i=1, \cdots, n} \sum_{j \in P_{i}} \delta_{l_{j}} d_{i} x_{i j} \leq b_{l} \quad \forall 1=1, \ldots, m \in$ links -capacity constraints (KNAPSACKS)

$$
x_{i j \in}\{0,1\} \quad \forall i=1, \ldots . n, j \in S_{i}
$$

The solution to the $l p$-relaxation using a subset of paths $S,\left(L P^{S}\right)$ is optimal for the $l p$-relaxation $L P^{P}$ if and only if the dual solution returned by the simplex method is feasible for the dual of $L P^{P}$. The dual constraint associated with variable $x_{i j}$ (corresponding to call $i$ using path $j$ ) is:

$$
\begin{aligned}
& y_{i}+d_{i} \sum_{l=1, \cdots, m} \delta_{l_{j}} z_{l} \geq r_{i}-d_{i} \sum_{l=1, \cdots, m} \delta_{l_{j}} c_{l} \\
& \text { or } d_{i}\left[\sum_{l=1, \cdots, m} \delta_{l_{j}}\left(z_{l}+c_{l}\right)\right] \geq r_{i}-y_{i}
\end{aligned}
$$

where $\delta_{l j}=1$ if link $l$ is in path $j ; 0$ otherwise.

We must determine whether a path $j$ for call $i$ is in the path set $P_{i}$, but not in our generated paths $S_{i}$, could improve the $L P^{S}$ solution. The column-generation subproblem associated with call $i$ is therefore:

$$
\begin{aligned}
& \min _{i}\left[\sum_{l=, \cdots, m} \delta_{l j}\left(z_{l}^{*}+c_{l}\right)\right] \\
& \text { subject to }: j \in P_{i}
\end{aligned}
$$

where $y_{i}^{*}$ and $z_{l}^{*}$ are the dual values returned by the simplex method in the current $l p$-relaxation. Thus, given a network with link weights $d_{i}\left(z_{l}^{*}+c_{l}\right)$, one finds the shortest path from the source node to the destination node of call $i$. If the solution to this shortest path problem has path lengths less than $r_{i}-y_{i}^{*}$, the current $l p$-solution is not optimal. Path $j$ should be added to the problem and the linear program re-solved. Since link weights are non-negative, a modified Dijkstra's (see Dial et al. 1979) algorithm can be used to find the shortest path(s) for the column-generation subproblem. We also note that by generating all shortest paths for all calls, we are likely to limit the number of times that the pricing routine will need to be called.

Our formulation of the column-generation subproblem differs from Parker and Ryan's in that we have a third set of dual variables corresponding to the dual prices on the cut constraints. Since our cuts are based on capacity constraints, and since capacity constraints relate to individual links, the cuts we generate add an additional cost to each link in the shortest path. It is therefore straightforward to augment the shortest path problem with these dual prices.

Specifically, if $K$ is the number of cuts so far generated, where $\pi_{i k}$ is the coefficient for call $i$ in cut $k$, and $\pi_{0 k}$ is the right-hand-side value of the cut constraint, then we denote the cut in the following form:

$$
w_{k}: \sum_{i=1, \cdots, n} \sum_{j \in S_{i} \subset P_{i}} \delta_{l_{i}} \pi_{i k} x_{i j} \leq \pi_{0 k} \quad \forall k \in K
$$

where $w_{k}$ will denote the dual variable corresponding to cut-constraint $k$. 
Then the column-generation subproblem associated with call $i$ is:

$$
\begin{aligned}
& \min d_{i} \sum_{l=1, \cdots, m}\left[\delta_{l_{j}}\left(z_{l}^{*}+c_{l}\right)\right]+\sum_{k=1}^{K} \delta_{l_{j}} \pi_{i k_{l}} w_{k}^{*} \\
& \text { subject to: } j \in P_{i}
\end{aligned}
$$

From this formulation of the shortest path problem, we see that the weights on each arc/link become $d_{i}\left(z_{l}^{*}+c_{l}\right)+\sum_{k=1}^{K} \pi_{i k_{l}} w_{k}^{*}$. As will be seen in the next section, the cuts we generate relate to the capacity of any link and are based on minimal covers for the capacity constraints (knapsacks) in the problem. Once a cut is generated for a call that uses that link, the coefficient $\pi_{i k_{l}}$ in the cover is included in that link's cost in the shortest path problem. If the solution to this shortest path problem is less than $r_{i}-y_{i}^{*}$, then the current $l p$ solution is not optimal and we have found another path to add to the problem.

\subsection{Generating Cuts}

Once the $l p$-relaxation is dual feasible, cutting plane constraints are generated and added to the $l p$-relaxation to better define the feasible integer solution space. When incorporated into a branch- and-cut procedure, these additional constraints reduce the solution space for the linear programming relaxation, while not cutting off any portion of the solution space that contains a feasible integer point. We used facial cuts similar to those described in Hoffman and Padberg (1991) and Crowder et al. (1983). We also exploit the fact that all paths for a call that use the same link have the same coefficient in the capacity constraint. This fact coupled with the fact that only one path can be chosen assures that the lifting coefficient on every such path is equal. Thus, one can find the lifting coefficient for every path of a given call by performing only one optimization! This result is a direct application of the theorem of Johnson and Padberg (1981) that provides a strong lifting procedure when one considers knapsacks with disjoint special-ordered sets.

Minimal-covers are generated based on the link capacity constraints and the set of special-ordered-set constraints:

$$
\begin{array}{ll}
\sum_{i=1, \cdots, n} \sum_{j \in S_{i}} \delta_{l_{j}} d_{i} x_{i j} \leq b_{l} & \text { for some link constraint l } \\
\sum_{j \in S_{i}} x_{i j} \leq 1 & \forall i=1 . . n
\end{array}
$$

In a manner similar to that described in Hoffman and Padberg (1991), we project out variables at both zero and at one. We then solve the knapsack problem with special-ordered sets only over the fractional variables. We then sequentially lift back any fractional variables not in the minimal cover, then all variables projected out at one, and finally those at zero.

Specifically, we consider some link $l$ and let $x_{i j}^{*}$ be the optimal solution to the current $l p$-relaxation. Define the following sets over the variables $x_{i j}$ that use link $l$ : $Q_{1}=\left\{x_{i j}^{*} \mid x_{i j}^{*}=1\right\}, Q_{0}=\left\{x_{i j}^{*} \mid x_{i j}^{*}=0\right\}$, and $Q_{F}=\left\{x_{i j}^{*} \mid 0<x_{i j}^{*}\right.$ $<1\}$. Also, let the set $T_{i}$ be the set of calls for which there is at least one $x_{i j}^{*}$ fractional. We then solve the following minimal-cover problem:

$$
\xi=\min \left\{\sum_{i \in T_{i}}\left(1-\sum_{i \in Q_{F}} x_{i j}^{*}\right) z_{i} \mid \sum_{i \in T_{i}} d_{i} z_{i}>b_{l}-\sum_{i \in Q_{1}} d_{i}, z_{i} \in\{0,1\}\right\}
$$

If $\xi<1$, then a valid inequality has been found which cuts off $x_{i j}^{*}$. We note that since all paths for this call that use this link require the same bandwidth, each such path has the identical coefficient value in the link-capacity constraint. Thus, if any path for this call is part of the minimal cover, then all paths for that call that use this link will also be part of the cover with the same cut (cover) coefficient. We can therefore perform the "lifting" of new paths that use a specific link into an existing minimal-cover constraint with virtually no additional computational effort.

In cut generation, we use strong lifting to tighten the problem. Once a minimal cover is found, we lift back any fractional variables that were not in the original cover, then lift back the variables that were projected out at one (see Wolsey, 1975 for details) and then lift back the variables at zero (see Padberg, 1975). Since these lifting procedures are sequential, different lifting orderings will generate different facets. We vary the order and generate up to five lifted minimal-cover inequalities for each link-capacity constraint.

We also lift newly generated paths (new columns in our $l p$-formulation) into the existing cuts because the special structure of this problem allows us to lift all paths for a call simultaneously. We can do this since the coefficient in the knapsack constraint is the same for all such paths. Thus, from the Johnson and Padberg result, we know that all such variables will have the same lifting coefficient. We keep track of whether a call has any paths that use a specific link. If, at some future time, we generate a new path for a call that uses a link previously unused for that call, we lift this new path into all cuts that are associated with that link. Thus, even as the dimension of the problem expands, we are able to maintain cuts based on the facial structure of this higher-dimensional polyhedron. 


\subsection{A lp-Based Heuristic}

A good feasible solution provides a lower bound to the problem. Parker and Ryan documented the value of having a good lower bound early. When they fed their algorithm the best known solution obtained by Anderson et al., problems were solved faster and/or the solution quality improved in most cases. We therefore created a $l p$-based heuristic which we call after every round of column and cut generation at the top of the tree. Within the tree, we call the heuristic before exiting the branch. Establishing a lower bound early on has several advantages. Variables can be fixed based on their reduced cost and the gap between the lp-optimal and ip-optimal solution-values. Fathoming rules can be applied earlier to prune nodes from the enumeration tree. Also, if the user requires a solution to within a specified tolerance, the entire algorithm can be terminated as soon as the lower and upper bounds differ by less than this tolerance.

The heuristic uses the current $l p$-relaxation in a procedure similar to the "Dive and Fix" algorithm described by Wolsey (1998). First, the heuristic fixes variables currently at 0 in the current $l p$-solution to 0 and variables at 1 to 1 . Then it sets all fractional variables with $l p$-solution values greater than 0.7 to 1 , or if none $>0.7$, it selects the fractional variable closest to 1 , fixes it to 1 , and re-solves the linear program. One of three cases will result: (1) the solution is integer-stop and save this solution if it is better than the previous best ip-solution; (2) the $l p$ is infeasible - stop; or (3) the linear program is feasible but the solution is not integer-repeat. We repeat, fixing variables and re-solving until the set of unfixed fractional variables is no more than 12 . Given that it is quite fast to completely enumerate the $2^{12}$ possibilities, we enumerate and choose the best of all feasible solutions.

At the top of the tree before initiating branching, we do a rigorous search for good $i p$-solutions easily available from the current $l p$-relaxation. We begin by fixing all variables at 0 to 0 and all variables at 1 to 1 . Then, each fractional variable greater than 0.5 is, in turn, is set to 1 . This $l p$ is solved and then the $l p$ heuristic described above is performed. Since the order in which fractional variables are set to 1 matters, repeating the $l p$-heuristic for each "candidate" variable $\left(x_{i j}^{*}>0.5\right)$ searches for $i p$-solutions from the most likely set of variables.

In our experiments the heuristic often found the optimal integer solution at the top of the tree or early within the branching tree. The rigorous heuristic at the top of the tree significantly improved the quality of the $i p$-solution in most cases. For comparison, we sent the lp-relaxations at the top of the tree to ILOG CPLEX 8.0
(2002) to solve the integer program. CPLEX often visited several hundred nodes before finding the optimal solution. Also, because we were not dependent on a branching rule to find good feasible solutions, we chose to use the node having the best (i.e., largest) objective function value; we call this a best-node search of the branching tree. Both Park et al. and Barnhart et al. use depth-first search in order to find feasible solutions.

\subsection{Branching}

We begin branching when no additional columns can be found that improve the $l p$-solution and we cannot find any cuts that cut off the current fractional value.

Our branching strategy is rather different from those used in the past. Park, Kang, and Park used traditional branching in their branch and bound algorithm, where each node in the tree opens two new branches; one forcing the selected $x_{i j}$ to 1 and the other to 0 . When $x_{i j}$ is forced to 1 , this is a very strong branch since all other paths for that call are set to zero. However, the other side of the branch, when $x_{i j}$ is set to 0 , is very weak. This branching scheme also impacted their pricing scheme. Often, they would generate a column that they would have to discard because it violated the branching constraints.

As described earlier, Barnhart et al., chose a branching strategy that tried to alter the objective value on both branches of the current node, and also assured that their shortest path calculations were not harmed by this branching scheme. We use a different branching rule, which also maintains these positive characteristics.

Since we already have a good $i p$-lower bound, we search the tree using the best node and use a hybrid branching strategy to select the branching variable and rule. The hybrid strategy uses one of three branching schemes based on which one indicates that it will alter the $l p$-bound on both new branches.

There are three possible branching rules:

(1) Choose a call $i$, and on one side, force this call into the solution and on the other side specify that this call will not be routed. $\left(\sum_{j \in S_{i}} x_{i j}=1\right.$, on one side; and $\sum_{j \in S_{i}} x_{i j}=0$ on the other side of the branch for some call $i$.)

(2) Choose a call $i$, and choose a capacitated link $l$ based on its dual price. On one branch, we specify that this call must use link $l$ and on the other side, 
we specify that this call cannot use link $l$. $\left(\sum_{j \in S_{i}, \partial_{l=1}} x_{i j}=1\right.$ on one side of the branch, and $\sum_{j \in S_{i}, \partial_{l=1}} x_{i j}=0$ on the other side for some call $i$ and link l.)

(3) Choose a path $i j$ for call $i$ that is fractional and branch on that path. $\left(x_{i j}=0\right.$ on one side of the branch, and $x_{i j}=1$ on the other for some call $i$ and path $j$.)

We have listed these branching rules in the order that they are applied in the algorithm. First, we check if there is slack on any of the routing constraints. If so, we branch on the call with the most slack. On one side of the branch, we change the constraint from an inequality to an equation and on the other side we force all paths for that call to be zero. This forces the linear program to decide whether or not to route the call.

When all routing constraints are tight, we identify the fractional variable $x_{i j}$ closest to 0.5 and its most capacitated link $l$. We then specify that either the call uses this link or it does not. This is equivalent to saying $\sum_{j \in S_{i}, \delta_{l_{j}}=l} x_{i j}=0$ on one branch and $\sum_{j \in S_{i}, \delta_{l j} \neq l} x_{i j}=0$ on the other. Thus, instead of only fixing one variable, we fix a set of variables on each side of the branching tree. To prevent paths that use this link (on the branch that does not allow such paths), we set the link weight for link $l$ to infinity. Similarly, if we wish to generate more paths that use this link, we can generate a shortest path from the source of call $i$ to link $l$, and then a shortest path from link $l$ to the destination of call $i$.

Finally, if the selected variable $x_{i j}$ is the only path for call $i$ that uses link $l$, then we do the traditional branching on variable $x_{i j}$.

\section{Importance of Generating All Optimal or Assuring That Every Branch Has a Feasible Linear Programming Solution}

We now discuss why one needs to generate all optimal solutions to the linear programming problem within the branch and bound tree. All rules for columngeneration procedures that we are aware of require only that one obtain an optimal solution at the top of the branching tree and that one use the normal fathoming strategies within the tree: fathom if (a) the linear programming problem is infeasible, (b) the linear programming problem produces a feasible integer solution, or (c) the linear programming solution provides an answer worse (or at least not better than) the best known integer solution.

We present a simple example that shows that using the standard rules (those currently published in virtually all) column generation papers is insufficient to guarantee optimality. Specifically, the literature states that one need only show that there is no column that improves the objective function (i.e., that the bound at the top of the tree is a proper bound). Once one has obtained this bound, one can then branch on a fractional variable and, if one then gets a proper bound at each node in the branching tree, when the tree is fathomed, one has obtained the optimal solution to the overall problem. We present a simple (seven variables, three constraints) set-partitioning problem that-when following these standard rules for branching and fathoming - does not generate the columns needed to find the optimal solution. Thus, the standard algorithm would conclude that there are no integer feasible solutions to a problem that has multiple optimal integer solutions.

All rules for column generation procedures that we are aware do not discuss this problem. In Barnhart et al. (1998) they mention that the initial restricted master problem must have a feasible $l p$-relaxation to ensure that proper dual information is passed to the pricing problem. However, they do not indicate that one needs a similar requirement throughout the tree. Thus, it is implied that once one has obtained an optimal linearprogramming solution at the top of the branching tree, one can use the normal fathoming strategies within the tree: fathom if (a) the linear programming problem is infeasible, (b) the linear programming problem produces a feasible integer solution, or (c) the linear programming solution provides an answer worse (or at least not better than) the best known integer solution. We note that adding artificial variables that ensure that a feasible solution to the LP relaxation at the top of the tree exists is not sufficient to not cut off optimal integer solutions throughout the tree. Our example will illustrate this point.

We present a simple example that shows that one needs to be very careful about fathoming rules for a column-generation approach. We present a simple (seven variables, three constraints) setpartitioning problem that shows that a column-generation phase must take place at each node of the branching tree-even when the linear programming relaxation obtains an infeasible or integer solution. 


\subsection{Example:}

Consider a set-partitioning problem with three constraints and where the possible columns are: $(1,0,0)$, $(0,1,0),(0,0,1),(1,0,1),(1,1,0),(0,1,1)$, and $(1,1,1)$. Let the objective value for each column be equal to the sum of the ones in that column,. The respective objective function values are therefore $(1,1,1,2,2,2,3)$. Now all feasible solutions to this problem have objective value $=3$. Assume that one begins the column-generation process with the following three columns: $(1,1,0)$, $(1,0,1)$ and $(0,1,1)$. The linear program solution for this three column problem is $\mathrm{z}^{*}=3$ with a solution vector of $(1 / 2,1 / 2,1 / 2)$. Since the master problem has a feasible $l p$-relaxation, one has proper dual information at the top of the tree and there is no need to generate an artificial column. Now, within the column generation process, we find that all other columns have reduced cost of zero, so no column can improve the objective function value. We stop and decide to branch without generating any new columns. We therefore choose to fix one of these three variables (columns) to either one or zero and "branch." On each side of the branching tree, the linear optimization has no feasible solution and we conclude (incorrectly) that the problem has no feasible points! This is clearly false.

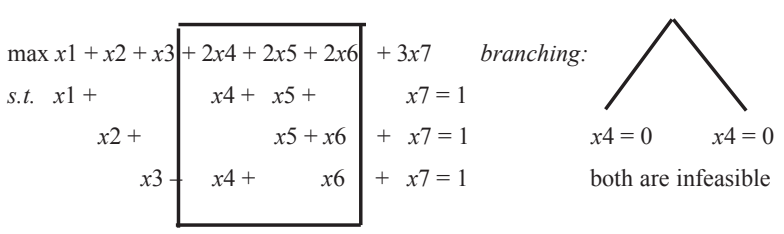

This simple problem highlights that the current literature has not completely specified what is needed to guarantee that important columns have not been ignored in a column-generation scheme. Instead of using the "normal" branch-and-bound fathoming rules, one must go through a column-generation phase at each node. In the case where solving the linear program provides an integer linear programming solution, one can use the dual prices from this solution to start the column-generation phase. In this case, the node will be fathomed only if, after column-generation, the solution remains integer. In the case where one obtains an infeasible solution, one needs to add artificial variables that make up a feasible solution (with the requisite high cost) in order to obtain the dual prices needed to begin the column-generation phase. If, after this columngeneration phase, the solution remains infeasible, the node be fathomed.

\section{Computational Results}

The software developed to test these ideas was written in $\mathrm{C}$ and runs on a $\mathrm{PC}$ in a Linux environment. CPLEX 8.0 is used to solve the $l p$ s using the simplex method with all preprocessing turned off. All problems were run on Dell OptimPlax PC with Red Hat Linux 8.0 operating system. ILOG CPLEX 8.0 (2002) Software Components library for Linux PC was used for solving all linear programming problems. We used none of the ILOG CPLEX integer programming components - thus, all branching rules, cutting planes, preprocessing and heuristic routines were written in $\mathrm{C}$ by the authors of this paper.

For our computational experiments, we used a set of 10 problems generated by US West and obtained by courtesy of Yuping Qiu. Since this set was also used by Laguna and Glover, and Anderson et al. in their work on the bandwidth-packing problem, we can draw comparisons to previous work. Laguna and Glover ran tests on this set of problems with and without link costs, while Anderson et al. show results only for the problems without link costs. IP1 through IP10 are the original problems and we denote the set without link costs with the letter $\mathrm{z}$ (e.g., IP1Z). We also ran tests on 14 problems (labeled DATA1 through DATA14) that were used by Barnhart et al. (2000) in their work. Among the two test bed sets, the problems range in size from 10 to 31 nodes, 15 to 61 links, and 20 to 93 calls. We present pictures of each of these problems in Appendix A.

We present our computational results in the three tables below. Table 1 presents our results. This table provides the number of columns and rows generated at the top of the tree and the linear programming relaxation (ZLP) and integer programming objective function value (ZIP) found at the top. We also indicate our success in solving each of these problems under the columns labeled "At Termination." 
Table 2. Computational results for BWP test sets without link costs

\begin{tabular}{|c|c|c|c|c|c|c|c|c|c|c|c|}
\hline \multirow[t]{3}{*}{ Problem } & \multicolumn{3}{|c|}{ Network/Call input } & \multicolumn{4}{|c|}{ At the top of the tree } & \multicolumn{4}{|c|}{ At termination } \\
\hline & Nodes & Links & Calls & Cols & Rows & $Z_{L P}$ & $Z_{I P}$ & $Z_{I P} @$ node & Cols & Rows & \\
\hline & & & & & & & & & & & Branches \\
\hline DATA1 & 14 & 16 & 35 & 68 & 73 & 6650.0 & 6580 & 6580@TOT ${ }^{\mathrm{a}}$ & 69 & 120 & 36 \\
\hline DATA2 & 24 & 24 & 68 & 87 & 95 & 7270.0 & 7270 & Solves at TOT & & & \\
\hline DATA3 & 29 & 61 & 70 & 693 & 349 & 28738.1 & 27550 & 28270@218 & 1778 & 2928 & $3000^{* b}$ \\
\hline DATA4 & 18 & 29 & 58 & 340 & 153 & 16826.4 & 16190 & 16210@87 & 488 & 600 & $3000^{*}$ \\
\hline DATA5 & 19 & 25 & 47 & 165 & 84 & 7790.0 & 7790 & Solves at TOT & & & \\
\hline DATA6 & 27 & 37 & 93 & 285 & 184 & 19053.8 & 18920 & 18970@426 & 308 & 614 & 862 \\
\hline DATA7 & 23 & 29 & 93 & 230 & 195 & 14109.1 & 13810 & 13880@131 & 307 & 465 & 716 \\
\hline DATA8 & 28 & 31 & 41 & 95 & 82 & 8825.0 & 8770 & 8770@TOT & 100 & 127 & 11 \\
\hline DATA9 & 24 & 42 & 87 & 445 & 198 & 21486.8 & 21360 & 21360@TOT & 700 & 452 & 404 \\
\hline DATA10 & 19 & 19 & 41 & 52 & 75 & 7810.0 & 7640 & 7640@TOT & 52 & 140 & 106 \\
\hline DATA11 & 14 & 16 & 23 & 43 & 53 & 6110.0 & 6110 & Solves at TOT & & & \\
\hline DATA12 & 27 & 36 & 81 & 283 & 160 & 13290.0 & 13290 & Solves at TOT & & & \\
\hline DATA13 & 29 & 31 & 52 & 141 & 96 & 9020.0 & 9020 & Solves at TOT & & & \\
\hline DATA14 & 20 & 23 & 46 & 118 & 90 & 8004.4 & 7900 & 7900@TOT & 136 & 191 & 86 \\
\hline IP1Z & 10 & 16 & 20 & 133 & 117 & 7783.6 & 7540 & 7540@TOT & 169 & 617 & 116 \\
\hline IP2 & 21 & 39 & 20 & 208 & 65 & 2100.0 & 2100 & Solves at TOT & & & \\
\hline IP3Z & 31 & 42 & 50 & 414 & 221 & 14004.3 & 13570 & 13710@1284 & 669 & 2817 & $3000^{*}$ \\
\hline IP4Z & 10 & 15 & 20 & 154 & 80 & 3035.7 & 2925 & 2955@38 & 158 & 548 & 114 \\
\hline IP5 & 16 & 22 & 20 & 194 & 108 & 2426.2 & 2295 & 2395@58 & 208 & 252 & 58 \\
\hline IP6Z & 17 & 26 & 30 & 263 & 130 & 9319.2 & 8830 & 9010@280 & 267 & 1002 & 280 \\
\hline IP7Z & 20 & 31 & 40 & 381 & 244 & 11235.0 & 11010 & 11160@316 & 428 & 2957 & 316 \\
\hline IP8Z & 12 & 18 & 36 & 291 & 130 & 12810.0 & 12460 & 12560@46 & 372 & 2915 & $3000^{*}$ \\
\hline IP9Z & 12 & 19 & 24 & 200 & 101 & 5780.0 & 5600 & 5780@4 & 200 & 107 & 4 \\
\hline IP10Z & 14 & 22 & 28 & 222 & 128 & 1000.9 & 970 & 970@TOT & 255 & 813 & 210 \\
\hline
\end{tabular}

TOT Stands for "Top of Tree," $Z_{I P} @$ node denotes the node at which the integer solution was found.

$\mathrm{b} * \quad$ Indicates the test problem was terminated early, before the optimal $i p$-solution was found and/or proven.

Table 3 shows the benefit of using a $l p$-based heuristic to find a good integer lower bound on the test problems. At the top of the tree before the rigorous $l p$-heuristic was implemented, the best ip-solution found was between $88.7 \%$ and $100 \%$ of the $l p$-solution, with the average for the test sets at $95.5 \%$. After the rigorous heuristic, the average was increased to $97.5 \%$ (ranging from $94.6 \%$ to $100 \%$ ). For test problems with the largest gap $\left(Z_{I P}\right.$ less than $95 \%$ of $Z_{L P}$ ), performing the rigorous heuristic reduced the gap by an average of 5.4 percentage points. At the top of the tree, the $i p$-solution found by the heuristic was the optimal solution in $54 \%$ of the problem sets however; it often required considerable branching to prove optimality. In four cases, we terminated the branching at 3000 nodes and could not prove optimality.

In Table 4, we present the prior best known solutions found by other researchers and compare these to the solutions that we obtained. 
Table 3. Quality of $i p$-solution at the top of the tree

\begin{tabular}{|c|c|c|c|c|c|}
\hline & $\begin{array}{c}Z_{L P} \text { at Top of } \\
\text { Tree }\end{array}$ & $\begin{array}{c}Z_{I P} \text { before } \\
\text { rigorous } \\
\text { heuristic }\end{array}$ & Tolerance & $\begin{array}{l}Z_{I P} \text { after } \\
\text { rigorous } \\
\text { heuristic }\end{array}$ & Tolerance \\
\hline DATA1 & 6650 & 6560 & $98.6 \%$ & 6580 & $98.9 \%$ \\
\hline DATA2 & 7270 & 7270 & $100 \%$ & & \\
\hline DATA3 & 28738.1 & 26170 & $91.1 \%$ & 27550 & $95.9 \%$ \\
\hline DATA4 & 16826.4 & 15540 & $92.4 \%$ & 16190 & $96.2 \%$ \\
\hline DATA5 & 7790 & 7790 & $100 \%$ & & \\
\hline DATA6 & 19053.8 & 18920 & $99.3 \%$ & 18920 & $99.3 \%$ \\
\hline DATA7 & 14109.1 & 13810 & $97.9 \%$ & 13810 & $97.9 \%$ \\
\hline DATA8 & 8825 & 8770 & $99.4 \%$ & 8770 & $99.4 \%$ \\
\hline DATA9 & 21486.8 & 20790 & $96.8 \%$ & 21360 & $99.4 \%$ \\
\hline DATA10 & 7810 & 7640 & $97.8 \%$ & 7640 & $97.8 \%$ \\
\hline DATA11 & 6110 & 6110 & $100 \%$ & & \\
\hline DATA12 & 13290 & 13040 & $98.1 \%$ & 13290 & $100 \%$ \\
\hline DATA13 & 9020 & 9020 & $100 \%$ & & \\
\hline DATA14 & 8004.4 & 7900 & $98.7 \%$ & 7900 & $98.7 \%$ \\
\hline IP1Z & 7783.6 & 7530 & $96.7 \%$ & 7540 & $96.9 \%$ \\
\hline IP2 & 2100 & 2100 & $100 \%$ & & \\
\hline IP3Z & 14004.3 & 12420 & $88.7 \%$ & 13570 & $96.9 \%$ \\
\hline IP4Z & 3035.7 & 2770 & $91.2 \%$ & 2925 & $96.4 \%$ \\
\hline IP5 & 2426.2 & 2295 & $94.6 \%$ & 2295 & $94.6 \%$ \\
\hline IP6Z & 9319.2 & 8830 & $94.8 \%$ & 8830 & $94.8 \%$ \\
\hline IP7Z & 11235 & 10780 & $95.9 \%$ & 11010 & $98.0 \%$ \\
\hline IP8Z & 12810 & 12280 & $95.9 \%$ & 12460 & $97.3 \%$ \\
\hline IP9Z & 5780 & 5500 & $95.2 \%$ & 5600 & $96.9 \%$ \\
\hline IP10Z & 1000.9 & 920 & $91.9 \%$ & 970 & $96.9 \%$ \\
\hline
\end{tabular}

Note: Bold values are optimal $i p$-solutions found at the top of the tree. However, in several cases of these cases, the solution was not proved optimal until branching was complete. 
Table 4. Comparison of best known solutions

\begin{tabular}{|c|c|c|c|c|c|}
\hline & $\begin{array}{l}\text { Glover and } \\
\text { Laguna }\end{array}$ & $\begin{array}{l}\text { Anderson } \\
\text { et al. }\end{array}$ & $\begin{array}{l}\text { Parker and } \\
\text { Ryan (starting } \\
\text { with no ip } \\
\text { lower bound) }\end{array}$ & $\begin{array}{l}\text { Parker and } \\
\text { Ryan (starting } \\
\text { with } \\
\text { Anderson ip } \\
\text { lower bound) }\end{array}$ & Ours \\
\hline DATA1 & & 6580 & 6580 & 6580 & 6580 \\
\hline DATA2 & & 7270 & 7270 & 7270 & 7270 \\
\hline DATA $3^{b}$ & & 27990 & 27010 & 28330 & 28270 \\
\hline DATA4 & & 16190 & 16190 & 16210 & 16210 \\
\hline DATA5 & & 7790 & 7790 & 7790 & 7790 \\
\hline DATA6 & & 18950 & 17810 & 18970 & 18970 \\
\hline DATA7 & & 13840 & 13760 & 13880 & 13880 \\
\hline DATA8 & & 8770 & 8770 & 8770 & 8770 \\
\hline DATA9 & & 21360 & 21000 & 21360 & 21360 \\
\hline DATA10 & & 7640 & 7640 & 7640 & 7640 \\
\hline DATA11 & & 6110 & 6110 & 6110 & 6110 \\
\hline DATA $12^{b}$ & & $13330 *^{\mathrm{a}}$ & 13230 & $13330^{*}$ & 13290 \\
\hline DATA13 & & 9020 & 8950 & 9020 & 9020 \\
\hline DATA14 & & 7900 & 7900 & 7900 & 7900 \\
\hline IP1Z & 7540 & 7540 & & & 7540 \\
\hline IP2 & 2100 & 2100 & & & 2100 \\
\hline $\mathrm{IP} \mathrm{Z}^{\mathrm{c}}$ & 13550 & 13270 & & & 13710 \\
\hline IP4Z & 2955 & 2885 & & & 2955 \\
\hline IP5 ${ }^{\mathrm{c}}$ & 2345 & 2365 & & & 2395 \\
\hline IP6Z & 9010 & 9010 & & & 9010 \\
\hline IP7Z & 11000 & 11160 & & & 11160 \\
\hline $\mathrm{IP}_{\mathrm{Z}} \mathrm{d}^{\mathrm{d}}$ & 12810 & 12810 & & & 12560 \\
\hline IP9Z & 5780 & 5780 & & & 5780 \\
\hline IP10Z & 970 & 970 & & & 970 \\
\hline
\end{tabular}

a * Indicates that prior work reported an incorrect optimal solution for problem DATA12. Anderson reported a solution of 13330. Having generated all feasible solutions via an enumeration procedure, we believe that no such solution exists. We believe that the best solution to this problem is 13290 .

b We also note that within our search-tree, which was truncated at 3000 nodes, the best solution found was 28270 for problem DATA3. We could,not totally enumerate all feasible columns as we did in DATA12 because we ran out of memory after generating only the columns for the first call. However, when generating 250000 columns for each call and sending this set of columns to ILOG's optimization code CPLEX, the code found 36 the solution 28330. thereby confirming that the Parker and Ryan solution of 28330 is feasible and the best solution known to date.

c We note that we have found better solutions to IP3Z and IP5 than had previously been reported.

d For problem IP8Z, we could not find the optimal solution within our 3000-node limit. We did confirm that the solution of 12810 is optimal through a complete enumeration procedure.

\section{Conclusions}

In this paper, we have combined the strengths of both column generation and cut generation, exploiting the special structure of the bandwidth-packing problem. It is the first paper to use the strength of special-ordered sets to strengthen minimal-cover cuts within a columngeneration setting, and we perform complete lifting. In addition, we have implemented a dynamic branching strategy that works well with our pricing algorithm. This new branching strategy fixes many variables within a single branch by again exploiting the special struc ture of the problem. We have also incorporated a linearprogramming based heuristic that seems to find very good solutions to the problem at the top of the tree. This process is especially important when one is not trying to prove optimality, but rather get measurably good integer solutions quickly. This heuristic is usable 
throughout the tree and can therefore use all of the information that the column generation, cutting planes and branching have provided. The use of this overall algorithm allowed us to find better integer solutions to certain problems than were previously known, allowed us to prove optimality in certain problems, and also showed some of the issues in implementing both column generation and cut generation within a single software package. If dual prices associated with cut constraints are not included in the pricing problem for column generation then columns that were previously generated are likely to be generated again, increasing run time. Finally, we provide a small example that shows that proving optimality to a column-generation code is far more difficult than previously imagined. Specifically, the linear program must not only be solved to proven optimality, but one must also generate all alternative solutions to ensure that we do not overlook an optimal column.

\section{Appendix A. BWP Test Sets}

These network test sets are those referred to in Tables 2 through 4 in Sec. 4 .

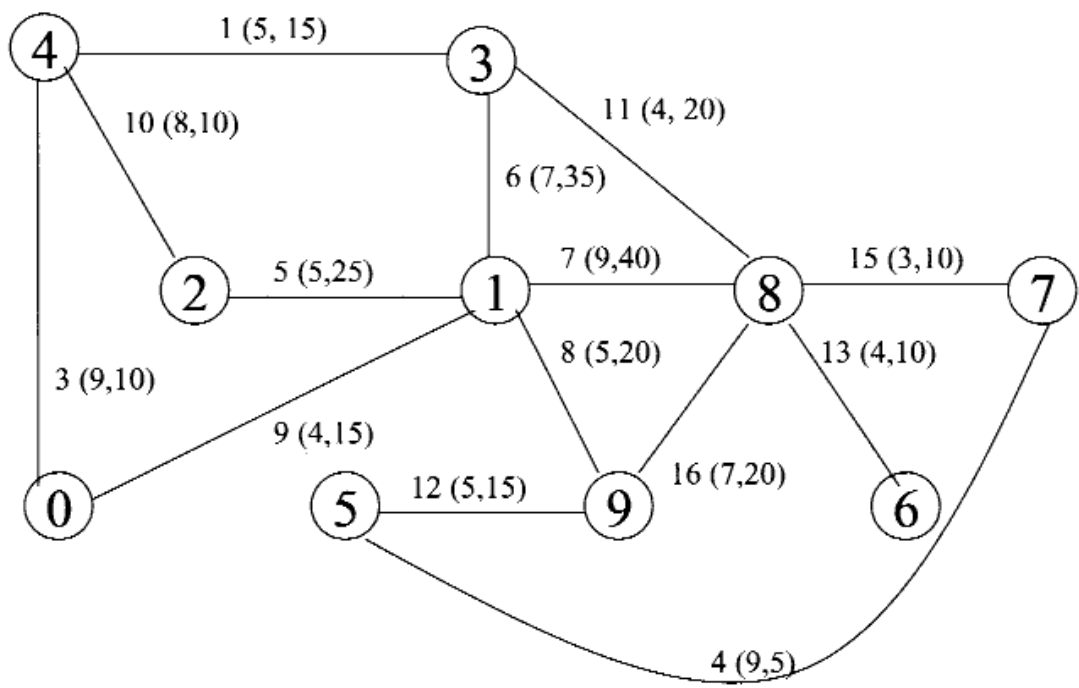

Fig. A1: Test Set IP1.

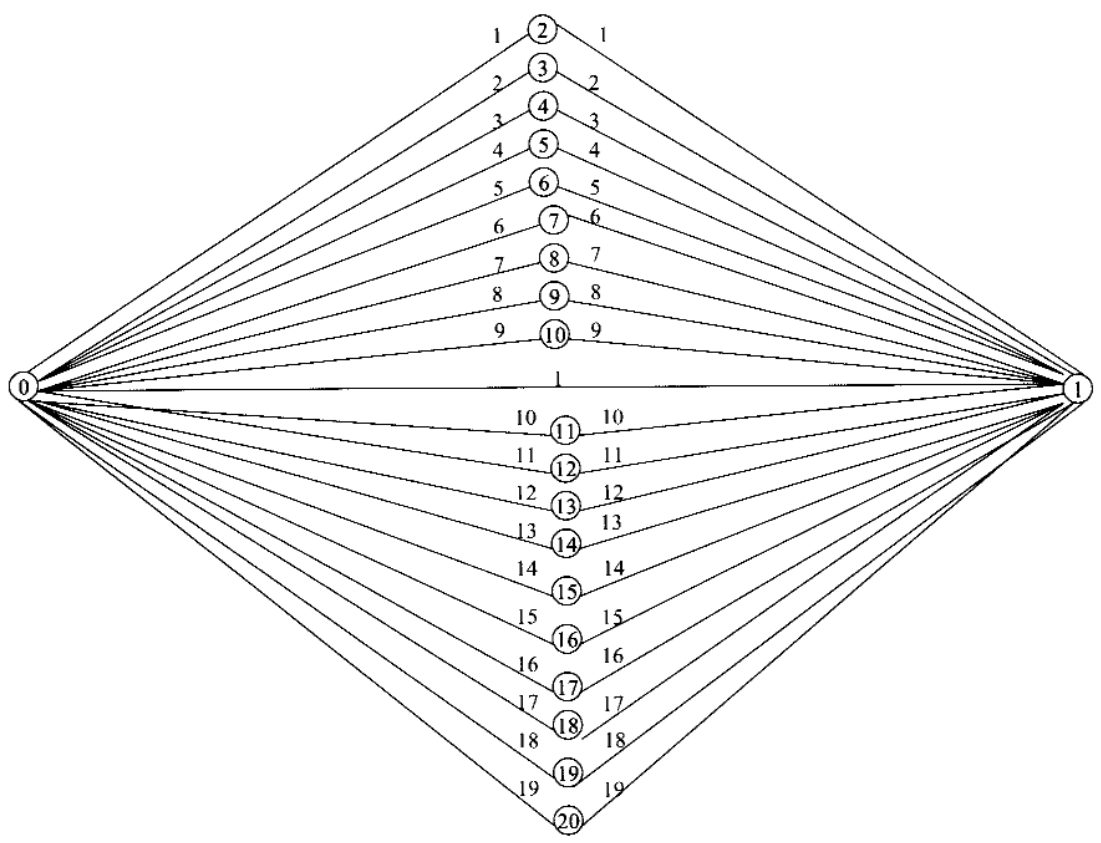

Fig. A2: Test Set IP2. 


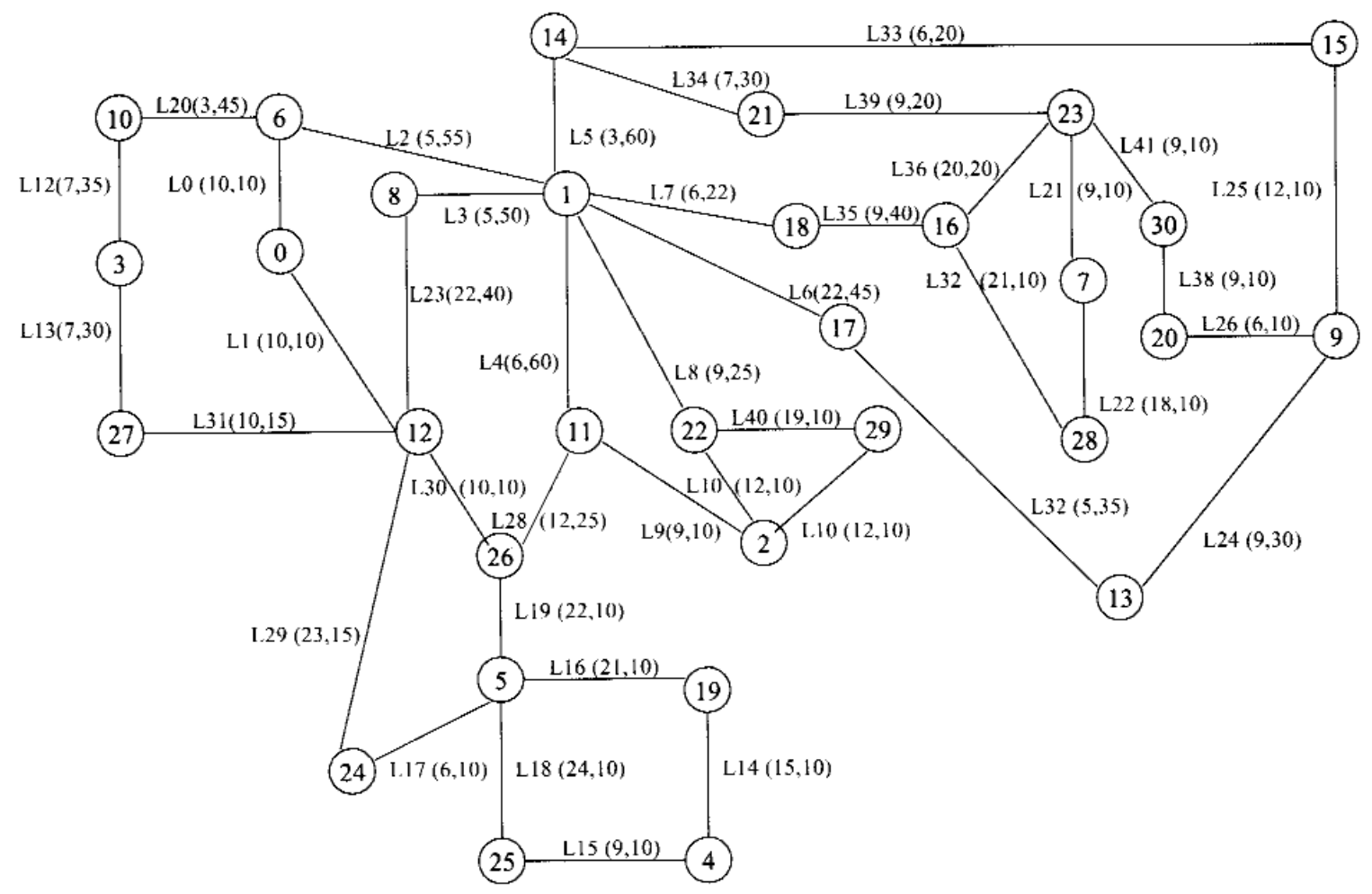

Fig. A3: Test Set IP3.

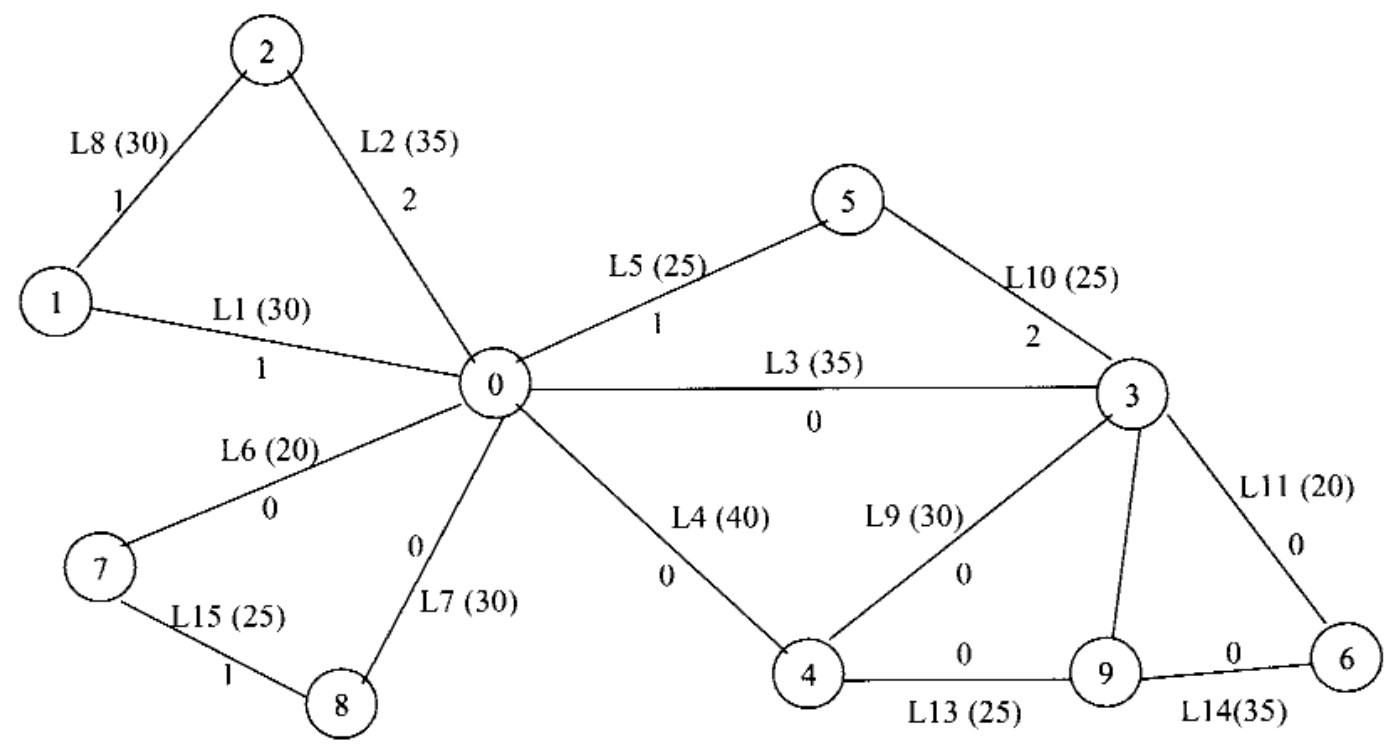

Fig. A4: Test Set IP4. 


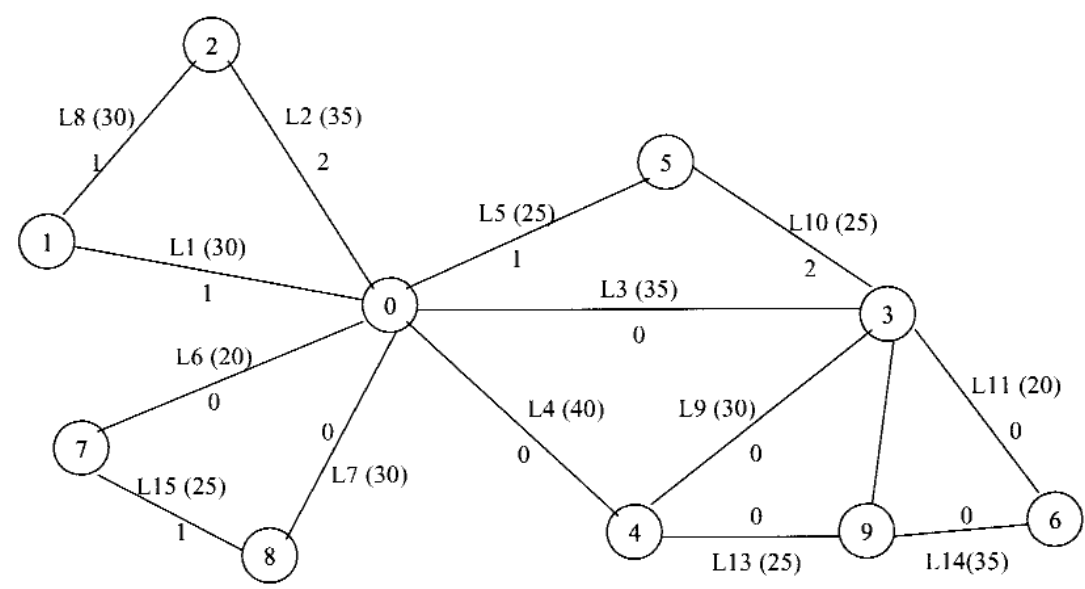

Fig. A5: Test Set IP5.

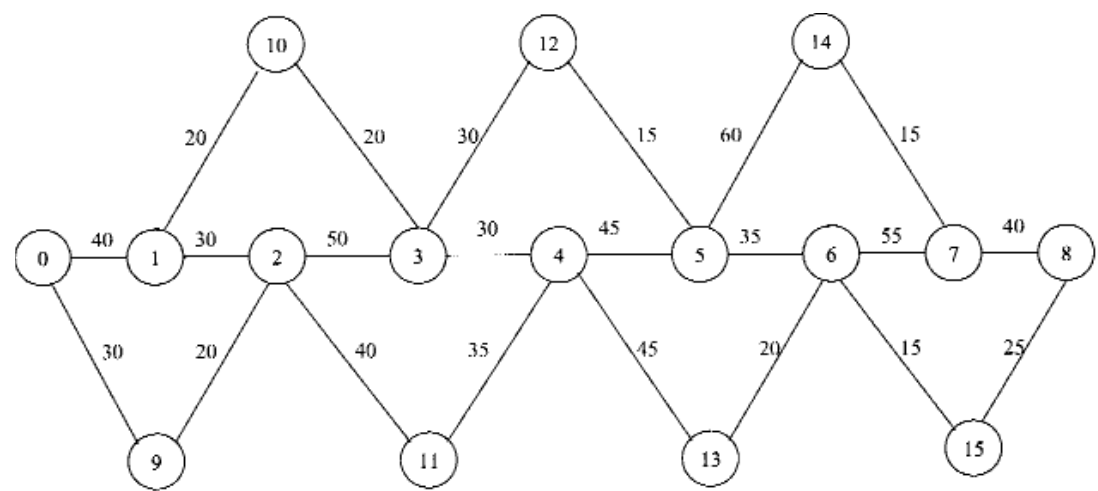

Fig. A6: Test Set IP6.

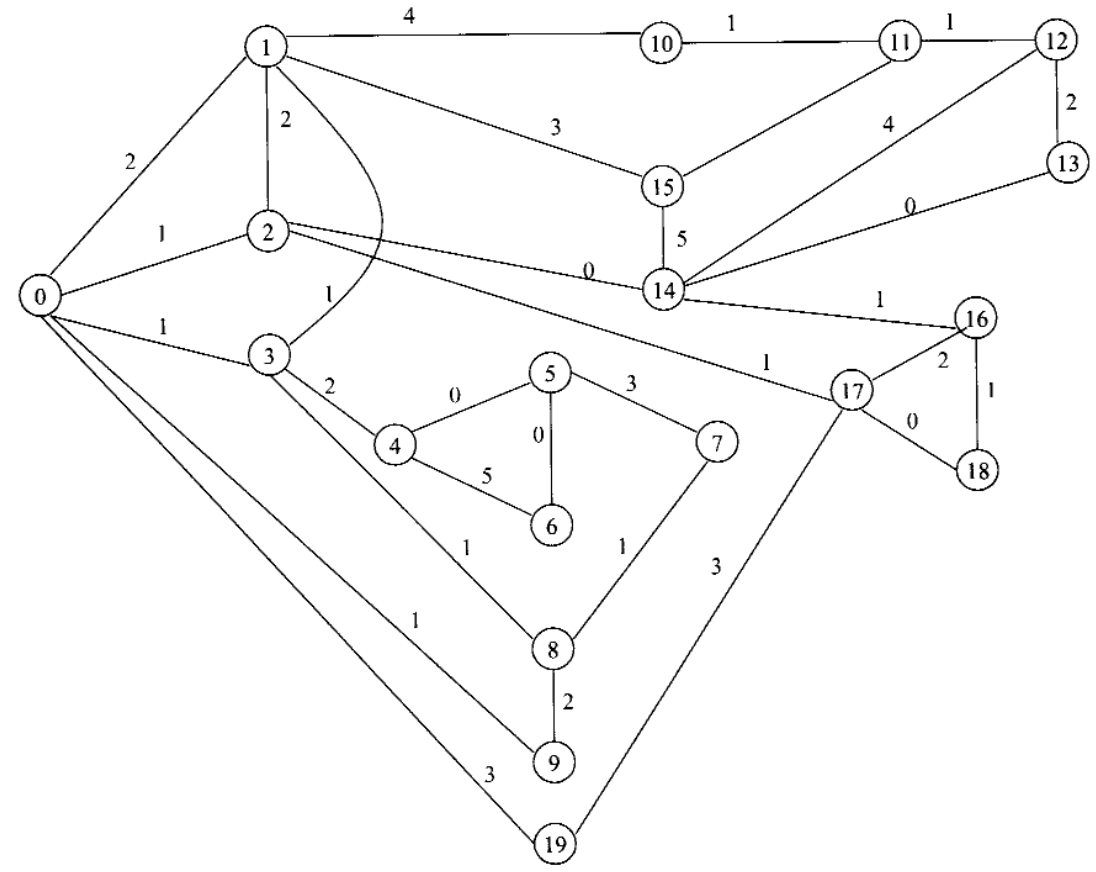

Fig. A7: Test Set IP7. 


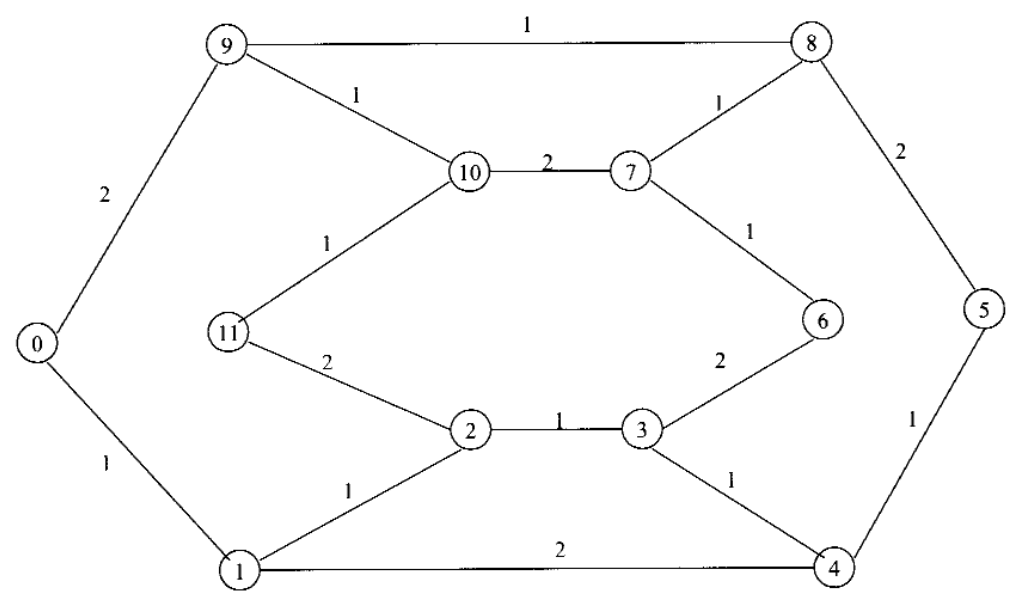

Fig. A8: Test Set IP8.

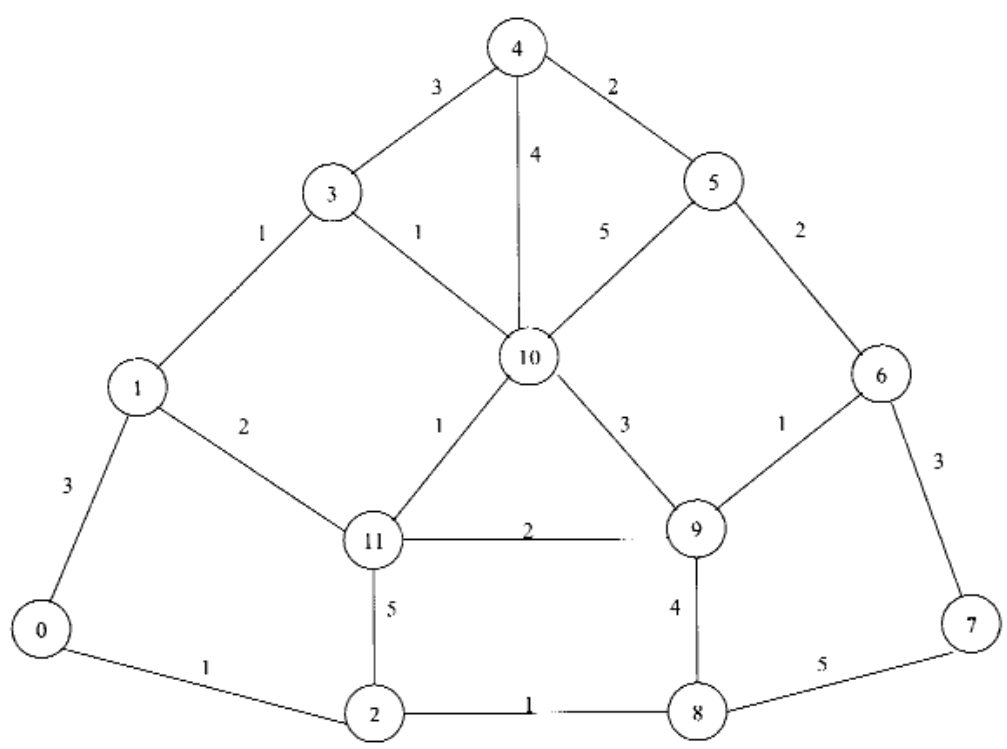

Fig. A9: Test Set IP9.

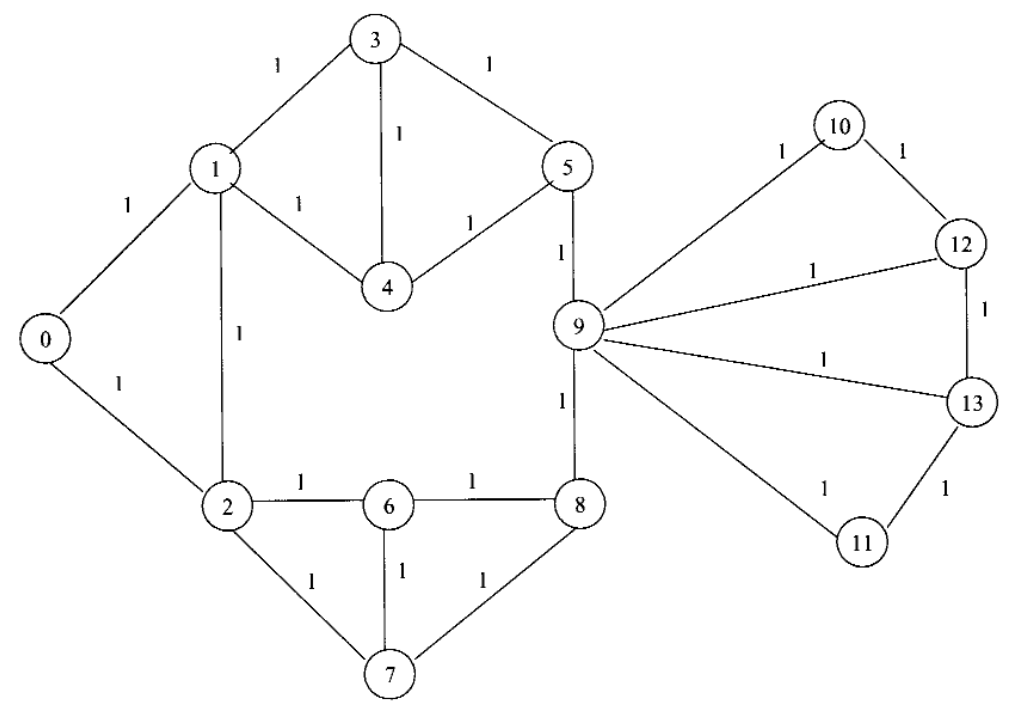

Fig. A10: Test Set IP10. 


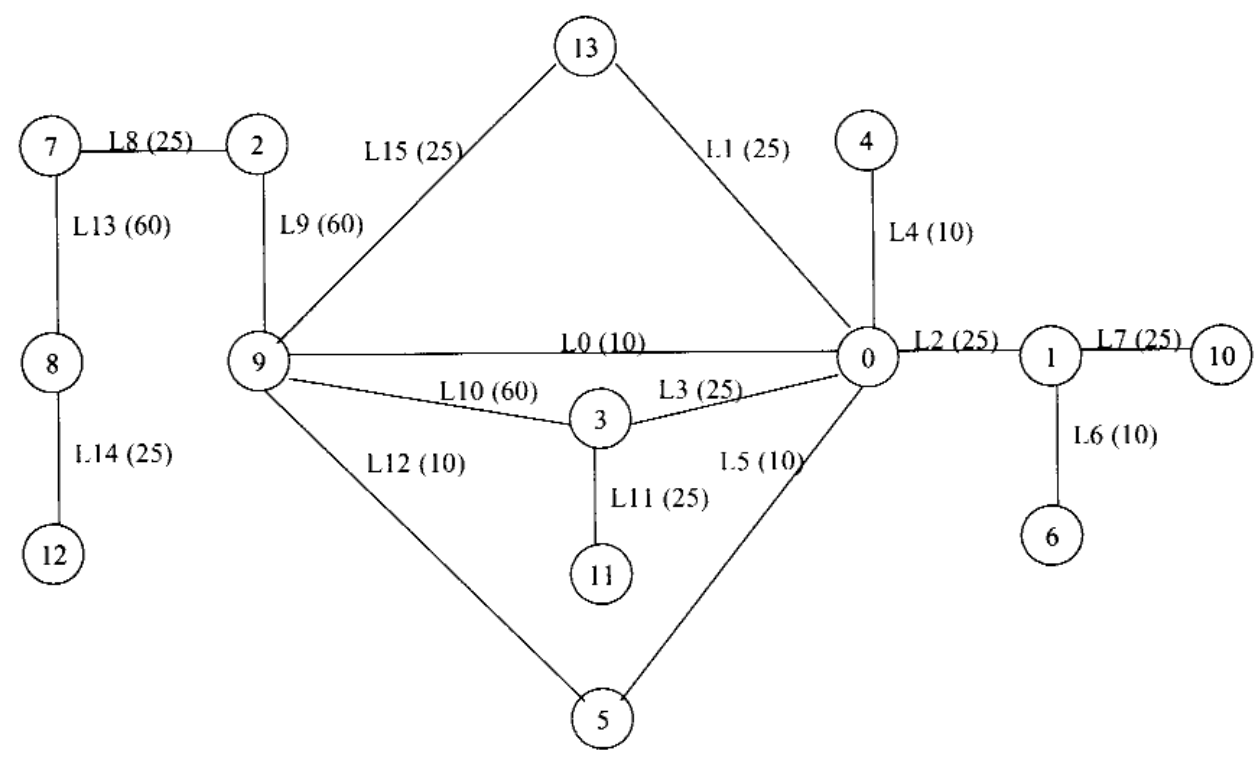

Fig. A11: Test Set DATA1.

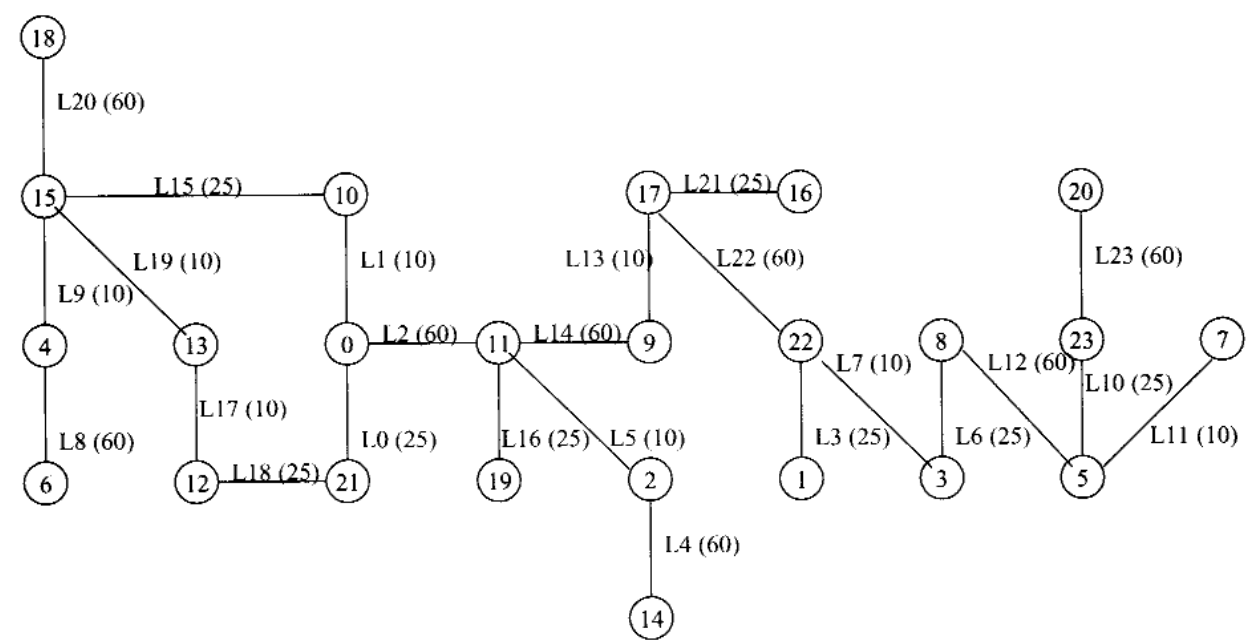

Fig. A12: Test Set DATA2. 


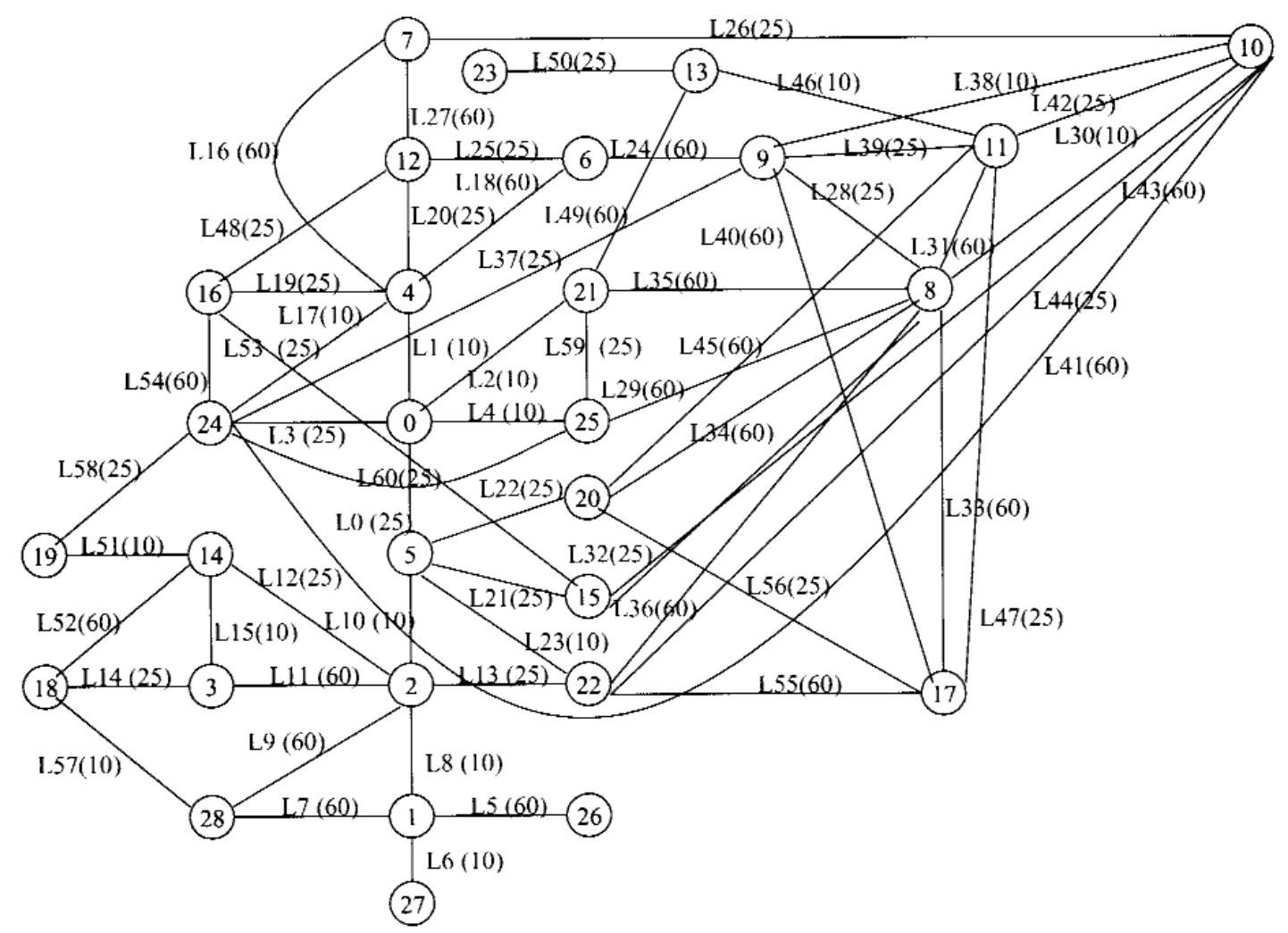

Fig. A13: Test Set DATA3.

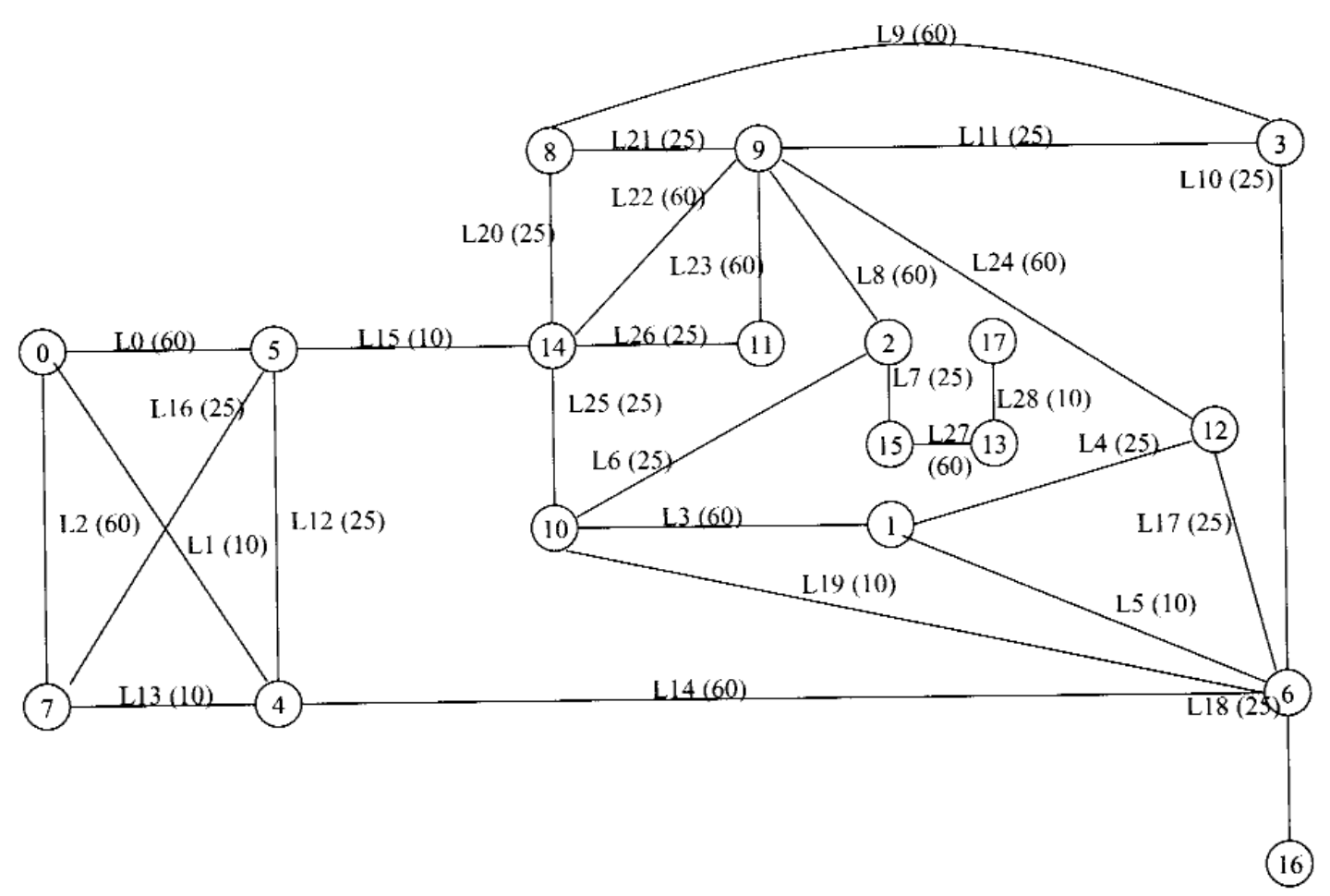

Fig. A14: Test Set DATA4. 


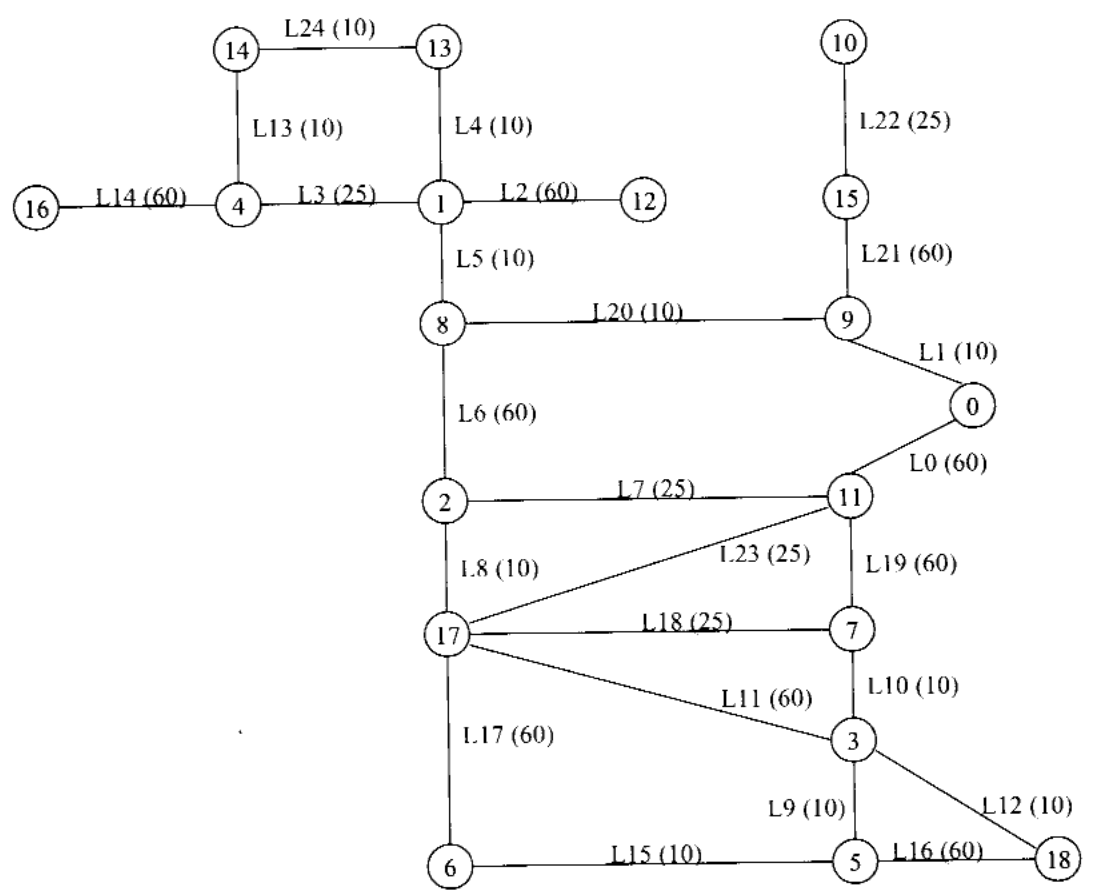

Fig. A15: Test Set DATA5.

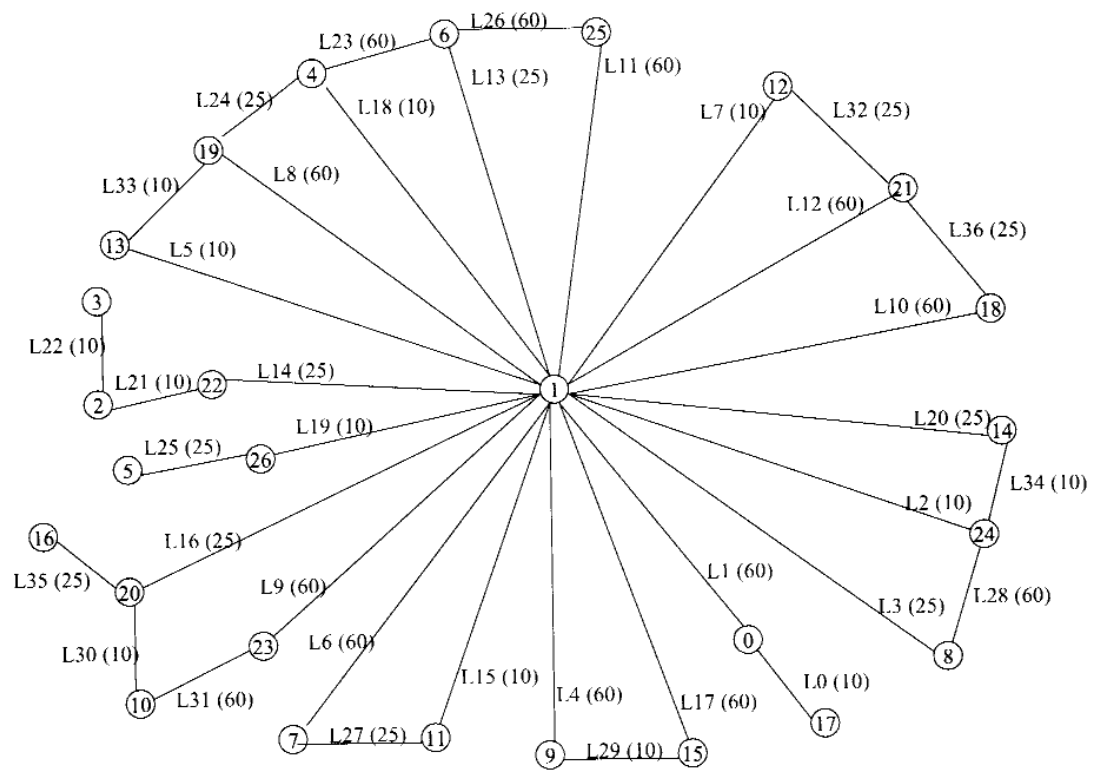

Fig. A16: Test Set DATA6. 


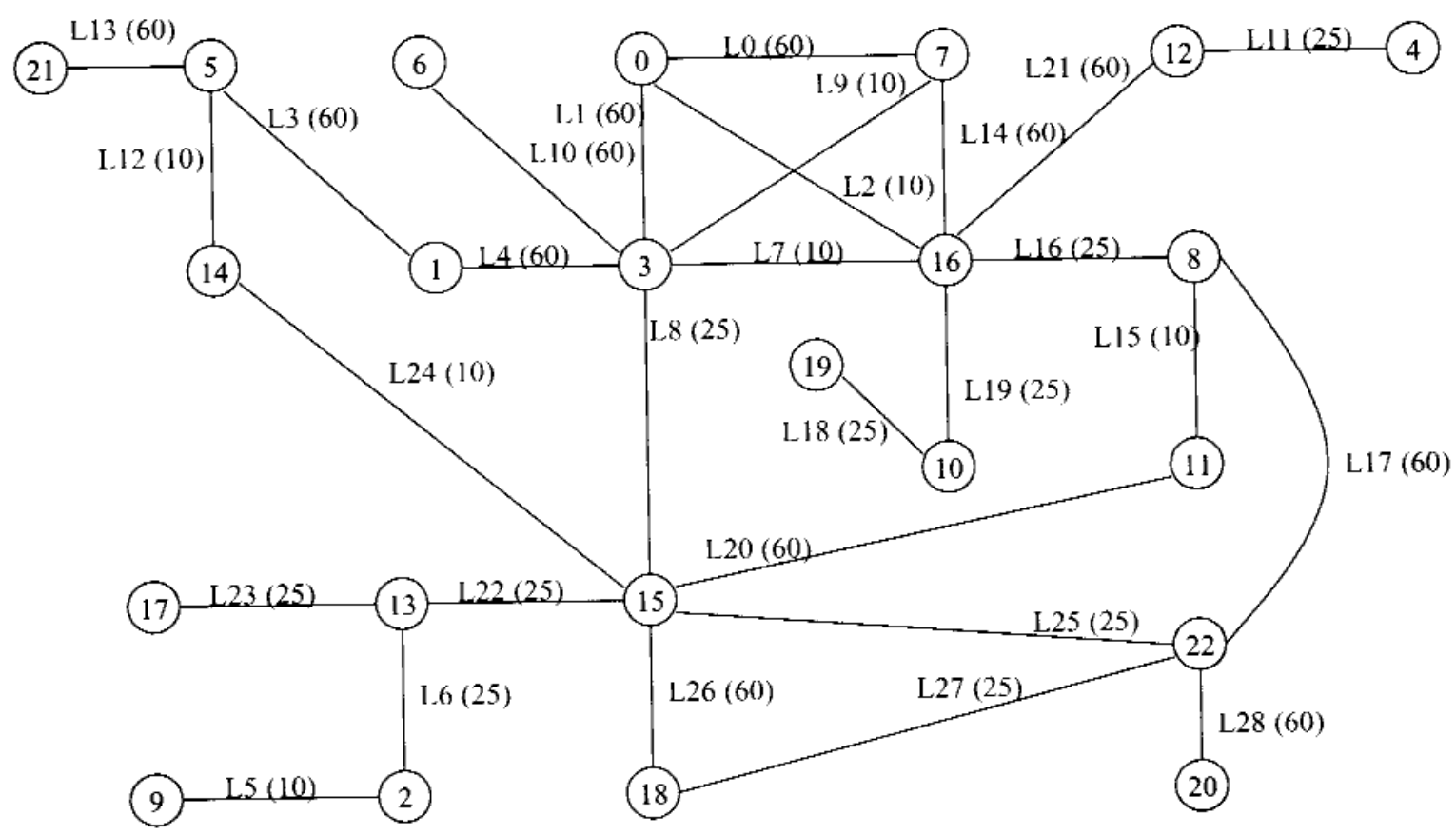

Fig. A17: Test Set DATA7.

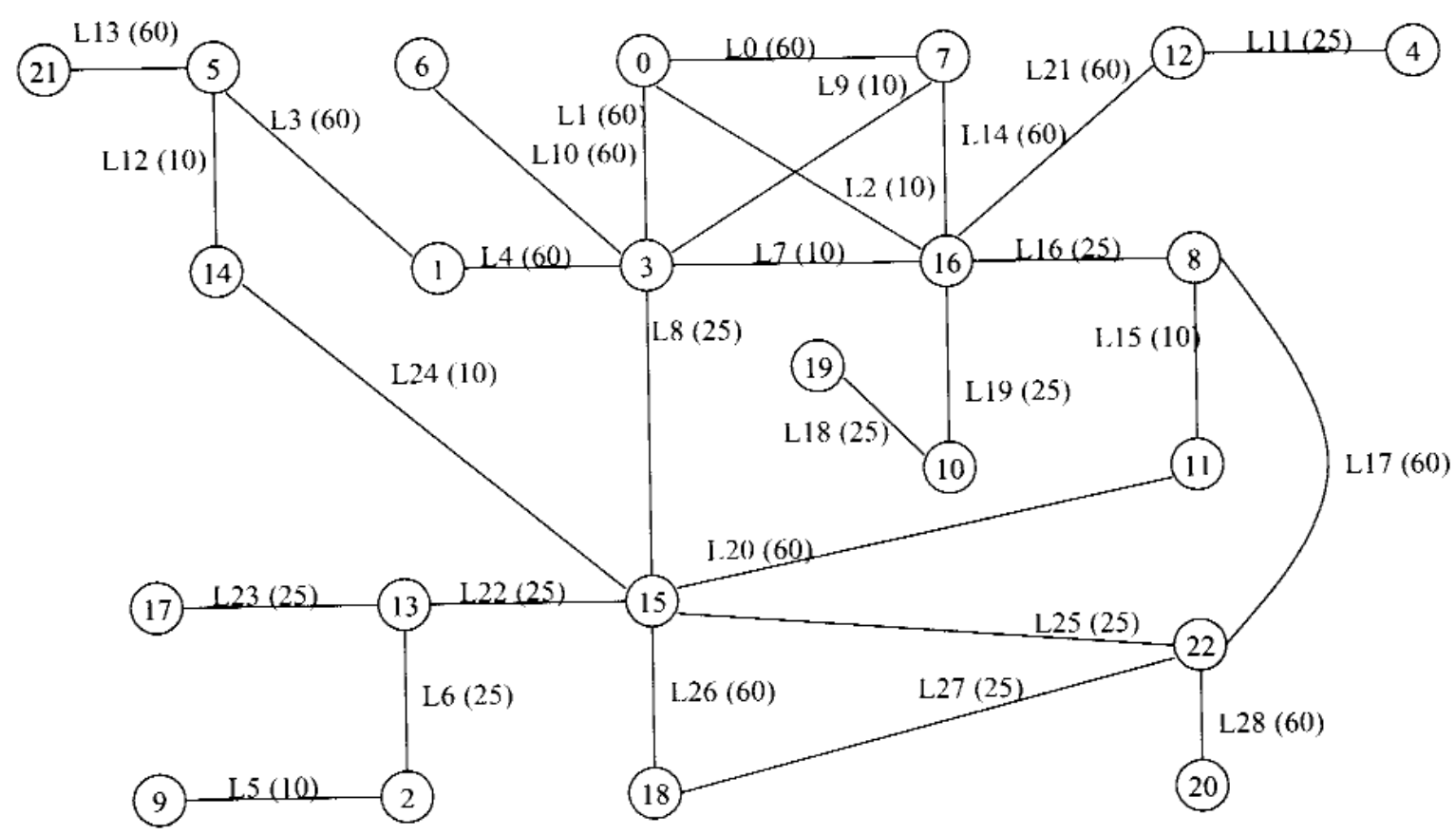

Fig. A18: Test Set DATA8. 


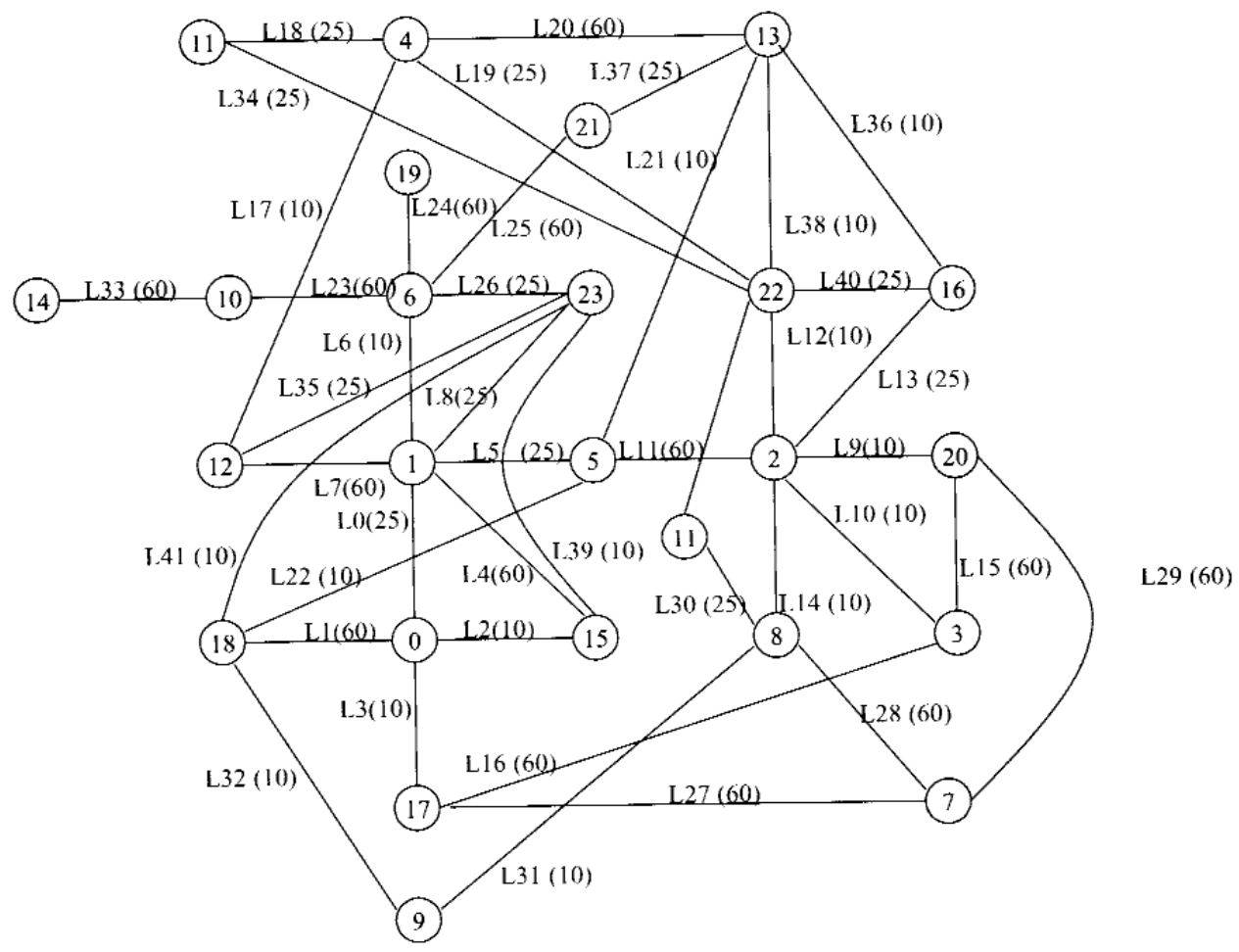

Fig. A19: Test Set DATA9.

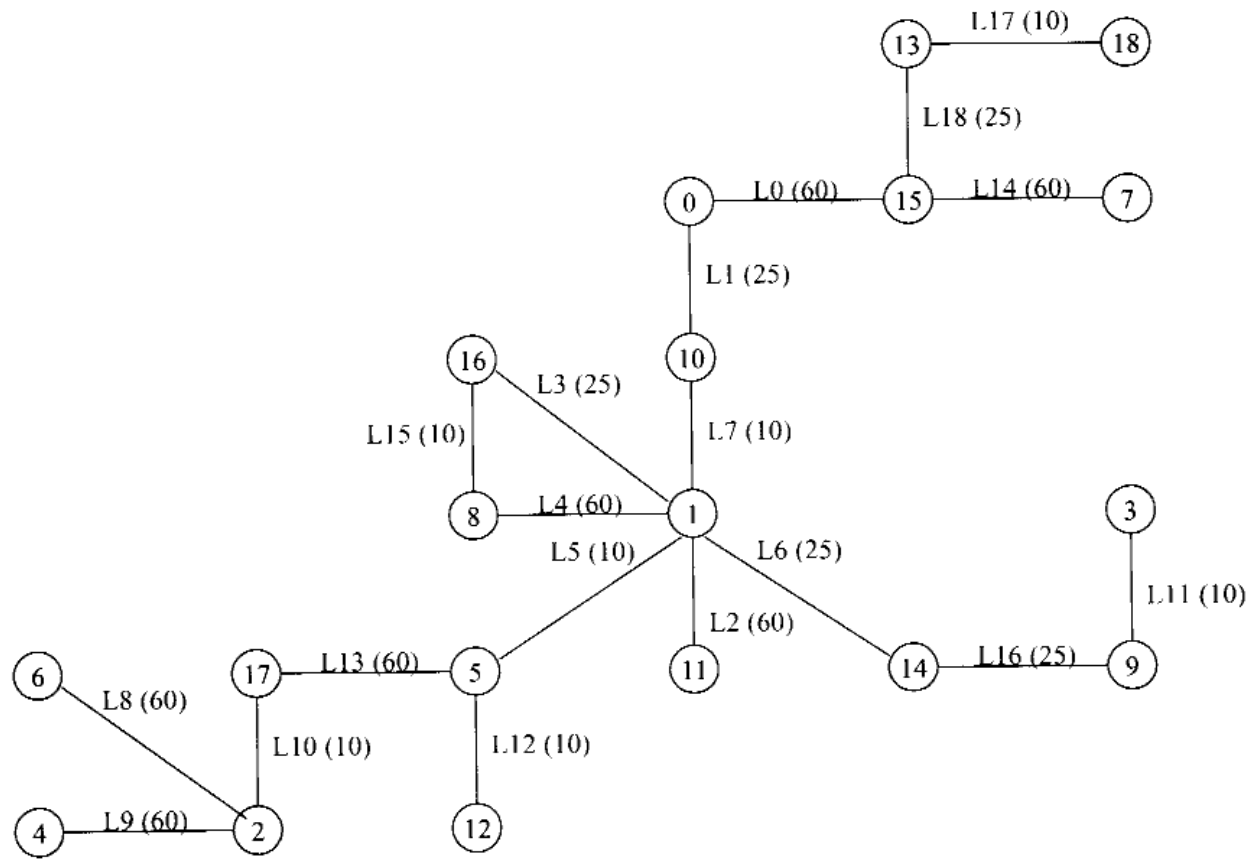

Fig. A20: Test Set DATA10. 


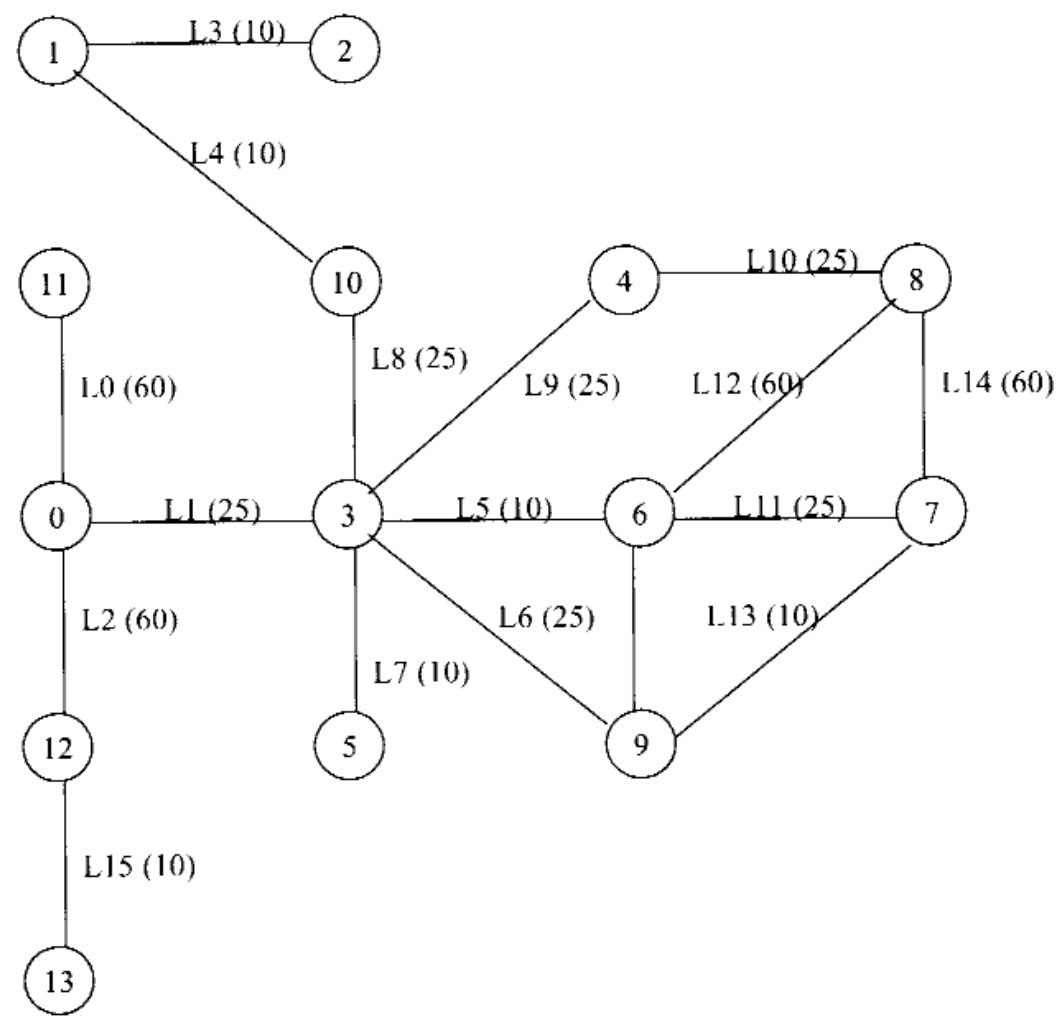

Fig. A21: Test Set DATA11.

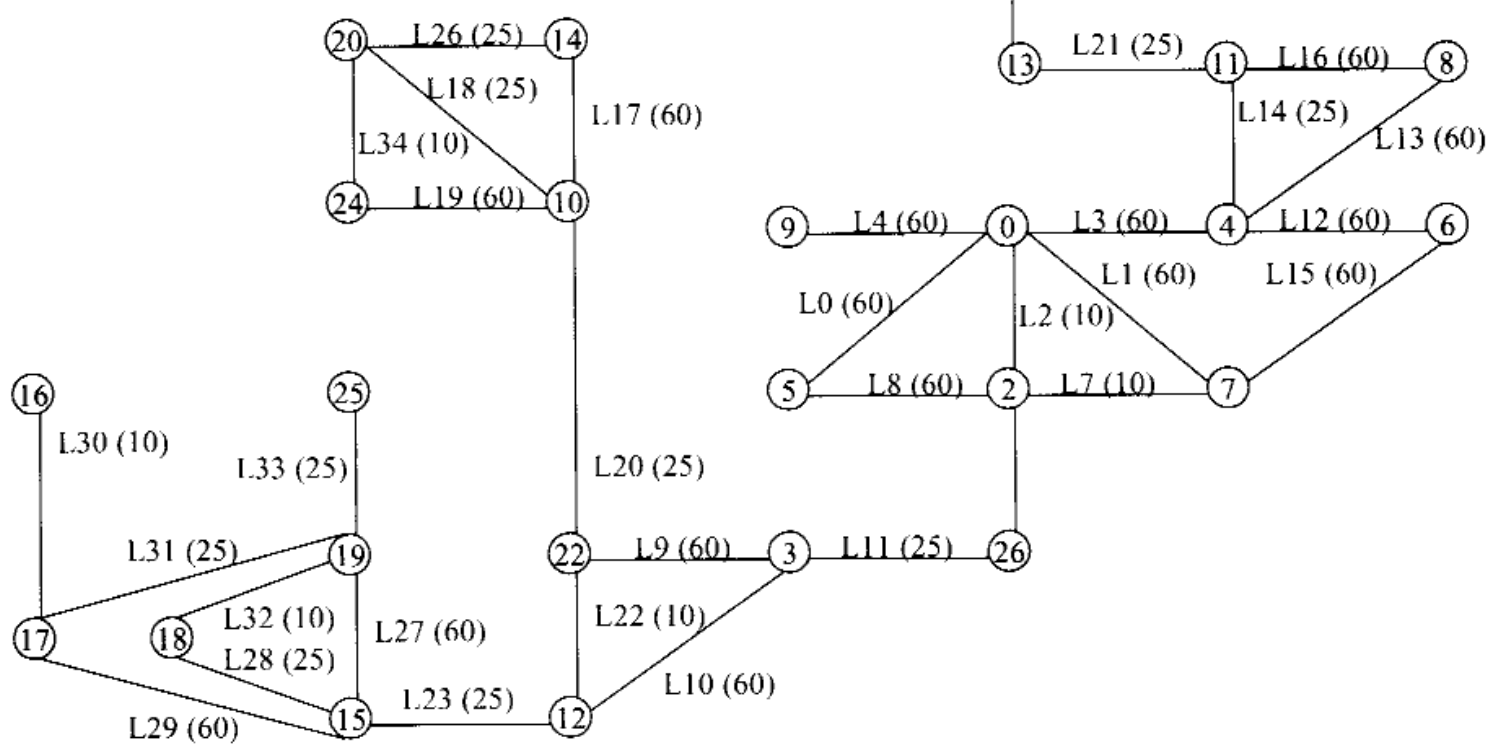

Fig. A22: Test Set DATA12. 


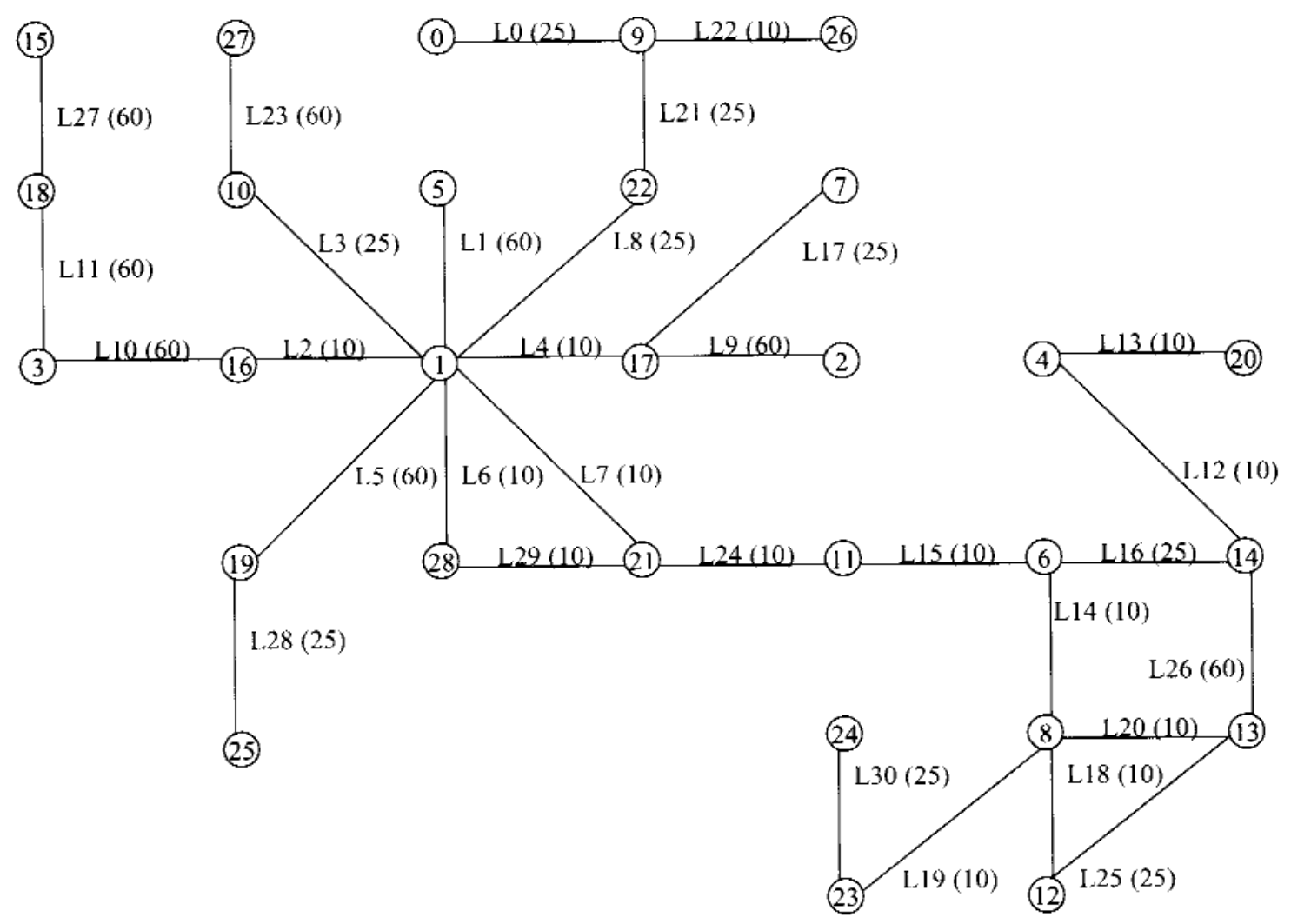

Fig. A23: Test Set DATA13.

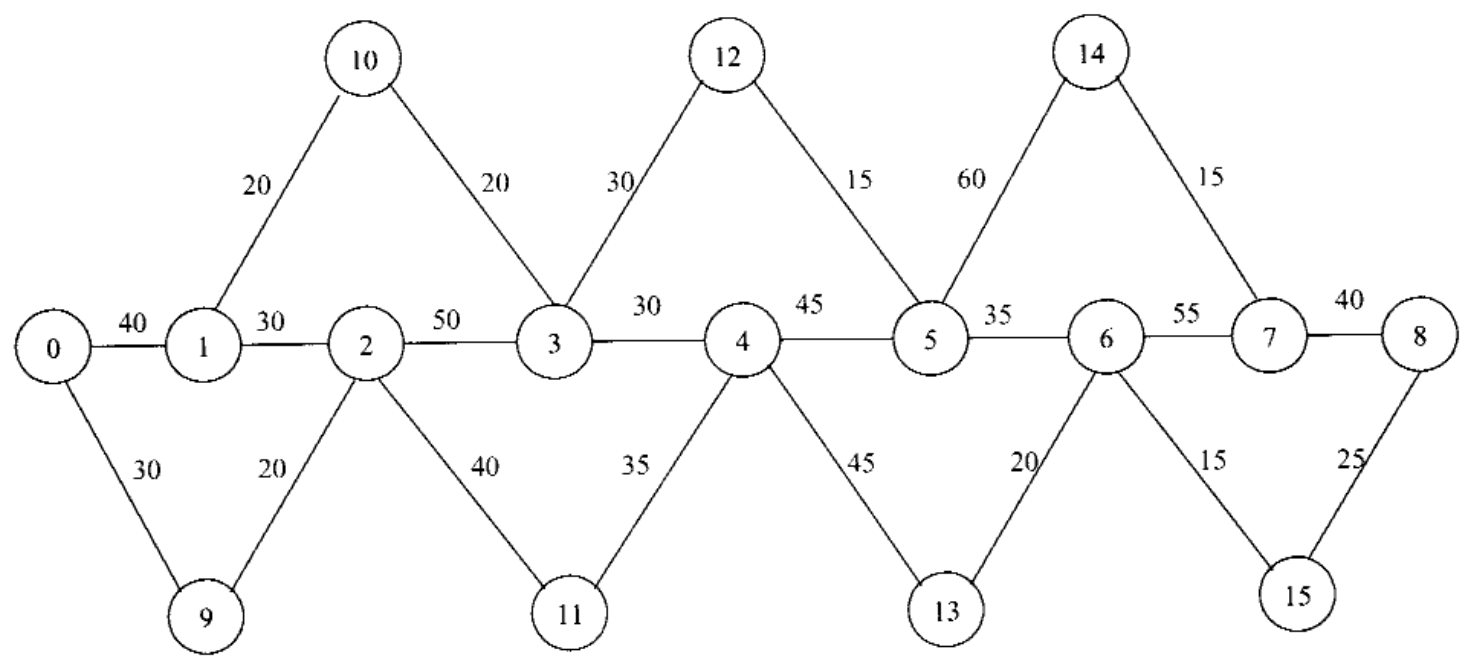

Fig. A24: Test Set DATA14. 


\section{Acknowledgements}

This research is partially supported by Office of Naval Research grant \# N00014-03-1-0192.

\section{References}

[1] A. Amiri and R. Barkhi, The multi-hour bandwidth-packing problem, Computers and Operations Research 27, 1-14 (1999).

[2] A. Amiri, E. Rolland, and R. Barkhi, Bandwidth packing with queueing delay costs: bounding and heuristic solution procedures, Eur. J. Oper. Res. 112, 635- 645 (1999).

[3] C. A. Anderson, K. F Jones, M. Parker, and J. Ryan, Path assignment for call routing: An application of tabu search, Annals of Oper. Res. 41, 301-312 (1993).

[4] C. Barnhart, C. A. Hane, and P. H. Vance, Using branch, price, and cut to solve origindestination integer multi-commodity Flow Problems, Oper. Res. 48, 318-326 (2000).

[5] ILOG CPLEX 8.0 Software component library for Linux PCs, December 2002.

[6] H. Crowder and M. W. Padberg, Solving large-scale zero-one linear programming problems, Oper. Res. 31, 803-834 (1983).

[7] L. A. Cox, L. Davis, and Y. Qiu, Dynamic anticipatory routing in circuit-switched telecommunications networks, in Handbook of Genetic Algorithms, Lawrence Davis ed., Van Nostrand Reinhold, New York (1991) pp. 124-143.

[8] R. Dial, F. Glover, D. Karney, and D. Klingman, A computational analysis of alternative algorithms and labeling techniques for finding shortest path trees, Networks 9, 215-248 (1979).

[9] M. Fischer, V. Sielen, G. Swinsky, and S. Wang, Topological network designs and analyses for the defense information system network, Military Oper. Res., 31-44 (1994).

[10] B. Gavish and K. Altinkemer, Backbone network design tools with economic tradeoffs, ORSA J. Comput. 2, 360-366 (1990).

[11] J. Geffard, A solving method for singly routing traffic demand in telecommunications Networks, Ann. Telecommun. 56, 1-10 (2001).

[12] W. Gu, G .L. Nemhauser, and M. W. P. Savelsbergh, Lifted cover inequalities for 0-1 integer programs I: Computation, Report COG 95-02, Industrial and Systems Engineering, Georgia Institute of Technology, Atlanta GA (1995).

[13] K. Hoffman and M. W. Padberg, Improving the representation of zero-one linear programs for branch-and-cut, ORSA J. Comput. 3, 121-134 (1991).

[14] E. Johnson and M.W. Padberg., A note on the knapsack problem with special-ordered sets', Oper. Res. Lett. 1, 18-22 (1981).

[15] M. Laguna and F. Glover, Bandwidth packing: A tabu search approach, Management Sci. 39, $492-500$ (1993).

[16] M. W. Padberg, A note on 0-1 programming, Oper. Res. 23, 833-837 (1975).

[17] M. W. Padberg and G. Rinaldi, A branch-and-cut algorithm for the resolution of large scale symmetric traveling salesman problems, SIAM Rev. 33, 60-100 (1991).

[18] K. S. Park, S. Kang, and S. Park, An integer programming approach to the bandwidthpacking problem, Management Sci. 42, 1277-1291 (1996).

[19] M. Parker, and J. Ryan, A column-generation algorithm for bandwidth packing, Telecommun. Syst. 2, 185-195 (1994).

[20] E. Rolland, A. Amiri, and R. Barkhi, Queueing delay guarantees in bandwidth packing, Computers and Oper. Res. 26, 921-935 (1999).
[21] L. Wolsey, Faces for a linear inequality in 0-1 variables, Math. Program. 8, 165-178 (1975).

[22] L. Wolsey, Integer Programming Wiley Interscience Series in Discrete Mathematics and Optimization. John Wiley \& Sons, New York (1998) p. 264.

About the authors: Karla Hoffman is a Professor in the Systems Engineering and Operations Research Department of George Mason University. Christine Villa is Vice President, Information Technology of BRTRC, Inc. in Fairfax, Virginia. 